\title{
Virtual Compton scattering off a spinless target in AdS/QCD
}

\section{Cyrille Marquet, ${ }^{a}$ Claude Roiesnel $^{b}$ and Samuel Wallon ${ }^{c, d}$}

${ }^{a}$ Institut de Physique Théorique, CEA/Saclay, 91191 Gif-sur-Yvette, France

${ }^{b}$ Centre de Physique Théorique, École Polytechnique, CNRS, 91128 Palaiseau, France

${ }^{c} L P T$, Université d'Orsay, CNRS, 91405 Orsay, France

${ }^{d}$ UPMC Univ. Paris 06, faculté de physique, 4 place Jussieu, 75252 Paris Cedex 05, France

E-mail: cyrille.marquet@cea.fr,

Claude.Roiesnel@cpht.polytechnique.fr, Samuel.Wallon@th.u-psud.fr

ABSTRACT: We study the doubly virtual Compton scattering off a spinless target $\gamma^{*} P \rightarrow$ $\gamma^{*} P^{\prime}$ within the Anti-de Sitter(AdS)/QCD formalism. We find that the general structure allowed by the Lorentz invariance and gauge invariance of the Compton amplitude is not easily reproduced with the standard recipes of the AdS/QCD correspondence. In the softphoton regime, where the semi-classical approximation is supposed to apply best, we show that the measurements of the electric and magnetic polarizabilities of a target like the charged pion in real Compton scattering, can already serve as stringent tests.

Keywords: AdS-CFT Correspondence, Electromagnetic Processes and Properties 


\section{Contents}

1 Introduction 1

2 Virtual Compton amplitude off an unpolarized target 3

3 The soft-wall model $\quad 5$

4 Calculation of four-point functions in AdS/QCD $\quad 7$

5 On-shell Compton amplitude $\quad 9$

6 Deeply inelastic scattering $\quad 14$

$\begin{array}{lll}7 & \text { Virtual Compton scattering } & 16\end{array}$

$\begin{array}{lll}8 & \text { Bjorken scaling of the DVCS amplitude } & 17\end{array}$

$\begin{array}{lll}9 & \text { Polarizabilities } & 19\end{array}$

$\begin{array}{ll}10 \text { Conclusion } & 21\end{array}$

A Explicit formulas in the hard-wall model 22

\section{Introduction}

The asymptotic conformal invariance of QCD has fostered a number of theoretical attempts to push the original AdS/CFT duality [1] beyond its conjectured domain of validity. The AdS/CFT correspondence postulates more generally a relation, through a well-defined set of prescriptions $[2,3]$, between weakly coupled string theories living in the bulk of an anti-de Sitter (AdS) space and strongly coupled conformally invariant field theories defined on its boundary. The efforts within the so-called AdS/QCD approach rely on the assumption that the AdS/CFT dictionary can still describe the strong coupling regime of a confining gauge theory like QCD, despite the breaking of conformal invariance, by computing correlation functions within a semi-classical field theory formulated in a fivedimensional AdS spacetime.

The various AdS/QCD models usually fall into two main categories. In the so-called "top-down", or "gauge/string", approach one sticks as much as possible to the original formulation of the AdS/CFT correspondence, and starts from a superstring theory living on $A d S_{5} \times M_{5}$ (where $M_{5}$ is some five-dimensional compact manifold) and tries to derive an effective theory which describes the low energy phenomena in QCD. The second, "bottom-up", or "gauge/gravity", approach puts aside the string-theoretic motivation of 
the correspondence and starts from a phenomenological Lagrangian in an appropriate five dimensional metric background which incorporates as much as possible the known properties of QCD. Contrary to some claims, the former approach is no more rigourous, in its present stage, than the latter.

The simplest way to break conformal invariance is by introducing a hard cut-off in AdS space which can be interpreted as an infrared mass scale of the gauge theory. The first "hard-wall" model [4] was able to generate a power-law scaling of glueball elastic scattering amplitudes at fixed angles. It is also possible to break conformal invariance softly through a background dilaton field which can be chosen [5] so as to reproduce the linear Regge behavior of the meson trajectories. Inside the bottom-up approach, it has proved possible to reproduce qualitatively within both hard-wall and soft-wall models the spectra of lowlying hadron states as well as various decay constants and coupling constants [6-9].

A frequent criticism claims that AdS/QCD models are nothing but some kind of "bag models" since quite a few other phenomenological models of QCD are able to reproduce the static parameters of hadronic states at the same level of accuracy. In fact such criticisms are pretty superficial because they ignore that AdS/QCD can incorporate in a coherent framework several of these models (vector meson dominance, $1 / \mathrm{N}$ expansion, sum rules, $\cdots$, see [10] for an up-to-date review of the pros and cons of the AdS/QCD approach).

Moreover the AdS/QCD models can also be used to study in a completely relativistic Lorentz invariant manner dynamical non-perturbative aspects like hadronic form factors or structure functions. In particular one can expect that conformal symmetry is most relevant for describing the electromagnetic interactions of hadrons, since the photon is a massless particle. ${ }^{1}$ A very important process that reveals the internal structure of hadrons is the deep inelastic scattering (DIS) of a highly energetic lepton off a hadron. This process was first investigated in a hard-wall model in [11]. Since then, there have been a number of related studies within the different flavors of AdS/QCD models [12-23]. However the semiclassical density of states of the hard-wall or soft-wall models with canonical dimensions does not agree [24] with the power-law behavior of the structure functions observed at high energy. It is presently far from clear how a correct partonic description of hadrons can emerge from the stringy corrections expected in the kinematical regime of DIS.

On the other hand the AdS/QCD models provide a low-energy description of the electromagnetic form factors which does agree with the dimensional counting rules of hadrons up to a few $\mathrm{GeV}$ [25-30]. Getting additional information about the electromagnetic structure of hadrons requires the study of four-point functions. There is one distinguished electromagnetic process which supplies all experimentally accessible information, namely Compton scattering. There has been a lot of theoretical work about Compton scattering. Strong interactions can significantly modify the amplitude but there is a low-energy theorem [31, 32] which guarantees that the Born contribution dominates near threshold. The two leading orders of an expansion of the real Compton scattering amplitude off a nucleon in terms of the frequency of the photon are entirely given in terms of the charge, mass and

\footnotetext{
${ }^{1}$ E.g. there is a dynamical $O(4,2)$ symmetry which is sufficient to determine completely the spectrum and eigenfunctions of the relativistic hydrogen atom.
} 
anomalous magnetic moment of the nucleon. For spinless targets like the pion, there is no linear term in the energy of photons. This theorem is based only on Lorentz invariance, gauge invariance and crossing symmetry. Quadratic corrections to the Born terms (electric and magnetic polarizabilities as well as generalized polarizabilities with virtual photons) are not specified by symmetry arguments alone and characterize non point-like elementary particles. Measuring these quantities allows to test the different models of strong interactions (see [33] for a review of the theoretical predictions on charged and neutral pion polarizabilities).

The purpose of this work is to study the Compton amplitude off a spinless target in the AdS/QCD formalism. We shall focus on the kinematical region where the photons are soft. The semi-classical approximation in the AdS/QCD duality should apply best in this region and there exist experimental measurements of the pion polarizabilities to compare with $[34,35]$. But we shall also study the kinematical region with one deeply virtual photon (DVCS) since it is straightforward to extract the corresponding structure functions in the Bjorken limit.

The plan of the paper is as follows. We shall begin by a brief summary of the standard lore about the general structure of the virtual Compton amplitude off an unpolarized target. Next we shall describe the generic soft-wall model we work with. In section 4 we shall explain how to calculate four-point functions in AdS/QCD and in section 5 we shall compute the Compton amplitude generated by the minimal coupling of a bulk scalar field with a bulk U(1) gauge field. We also compare our results with the recent hardwall calculation of [36]. In the subsequent section we shall make explicit the structure of this Compton amplitude in the deep inelastic region, and in section 7 we shall clarify its Lorentz and gauge-invariant structure in the DVCS kinematical region. Then we shall extract the corresponding structure functions in the Bjorken limit. Section 9 is devoted to the calculation of the polarizabilities of a spinless target. We shall comment on the implications of our results in the conclusion.

\section{Virtual Compton amplitude off an unpolarized target}

The amplitude of the virtual Compton scattering, $\gamma^{\star}\left(q_{1}\right)+A\left(p_{1}\right) \rightarrow \gamma^{\star}\left(q_{2}\right)+A\left(p_{2}\right)$, where $A$ is a spinless, or spin-averaged, hadron is defined through the off-forward matrix element of the time-ordered product of two electromagnetic currents,

$$
T_{\mu \nu}=i \int d^{4} x e^{i q \cdot x}\left\langle p_{2}\left|T\left\{J_{\mu}(x / 2) J_{\nu}(-x / 2)\right\}\right| p_{1}\right\rangle, \quad q=\frac{1}{2}\left(q_{1}+q_{2}\right) .
$$

In general, a two-to-two scattering amplitude depends on six independent kinematical invariants, namely the external virtualities $q_{1}^{2}, q_{2}^{2}, p_{1}^{2}, p_{2}^{2}$ and the usual Mandelstam variables

$$
s=\left(p_{1}+q_{1}\right)^{2}, \quad t=\left(p_{1}-p_{2}\right)^{2}, u=\left(p_{2}-q_{1}\right)^{2},
$$

obeying the constraint

$$
s+t+u=q_{1}^{2}+q_{2}^{2}+p_{1}^{2}+p_{2}^{2} .
$$


It is convenient, for calculating the Compton form factors, to choose $q_{1}, q_{2}$ and $p=p_{1}+p_{2}$ as the three independent momenta of the process. At most thirteen independent tensors can contribute to the Compton amplitude,

$$
\begin{aligned}
& g^{\mu \nu}, p^{\mu} p^{\nu}, q_{1}^{\mu} q_{1}^{\nu}, q_{2}^{\mu} q_{2}^{\nu}, q_{1}^{\mu} q_{2}^{\nu}, q_{2}^{\mu} q_{1}^{\nu}, p^{\mu} q_{1}^{\nu}, q_{2}^{\mu} p^{\nu}, p^{\mu} q_{2}^{\nu}, q_{1}^{\mu} p^{\nu}, \\
& \epsilon_{\mu \nu \rho \sigma} p^{\rho} q_{1}^{\sigma}, \quad \epsilon_{\mu \nu \rho \sigma} p^{\rho} q_{2}^{\sigma}, \quad \epsilon_{\mu \nu \rho \sigma} q_{1}^{\rho} q_{2}^{\sigma} .
\end{aligned}
$$

The antisymmetric tensors are parity-violating. Electromagnetic gauge invariance implies that

$$
q_{1}^{\mu} T_{\mu \nu}=T_{\mu \nu} q_{2}^{\nu}=0 .
$$

From the vanishing of the six coefficients of the linearly independent vectors, one can deduce five linearly independent conditions. Hence the most general spin-averaged, gaugeinvariant, and parity-conserving Compton amplitude has five independent form factors:

$$
\begin{aligned}
T^{\mu \nu}= & V_{1}\left(g^{\mu \nu}-\frac{q_{1}^{\mu} q_{1}^{\nu}}{q_{1}^{2}}-\frac{q_{2}^{\mu} q_{2}^{\nu}}{q_{2}^{2}}+q_{1}^{\mu} q_{2}^{\nu} \frac{\left(q_{1} \cdot q_{2}\right)}{q_{1}^{2} q_{2}^{2}}\right) \\
& +V_{2}\left(p^{\mu}-q_{1}^{\mu} \frac{\left(p \cdot q_{1}\right)}{q_{1}^{2}}\right)\left(p^{\nu}-q_{2}^{\nu} \frac{\left(p \cdot q_{2}\right)}{q_{2}^{2}}\right) \\
& +V_{3}\left(q_{2}^{\mu}-q_{1}^{\mu} \frac{\left(q_{1} \cdot q_{2}\right)}{q_{1}^{2}}\right)\left(q_{1}^{\nu}-q_{2}^{\nu} \frac{\left(q_{1} \cdot q_{2}\right)}{q_{2}^{2}}\right) \\
& +V_{4}\left(p^{\mu}-q_{1}^{\mu} \frac{\left(p \cdot q_{1}\right)}{q_{1}^{2}}\right)\left(q_{1}^{\nu}-q_{2}^{\nu} \frac{\left(q_{1} \cdot q_{2}\right)}{q_{2}^{2}}\right) \\
& +V_{5}\left(q_{2}^{\mu}-q_{1}^{\mu} \frac{\left(q_{1} \cdot q_{2}\right)}{q_{1}^{2}}\right)\left(p^{\nu}-q_{2}^{\nu} \frac{\left(p \cdot q_{2}\right)}{q_{2}^{2}}\right) .
\end{aligned}
$$

The form factors $V_{1}, V_{2}, V_{3}, V_{4}$ and $V_{5}$ can be readily identified as the coefficients of the tensors $g^{\mu \nu}, p^{\mu} p^{\nu}, q_{1}^{\nu} q_{2}^{\mu}, p^{\mu} q_{1}^{\nu}$ and $p^{\nu} q_{2}^{\mu}$ respectively. They are in general functions of the six independent scalar invariants. The off-shell Compton form factors are not directly measurable. The Compton form factors of on-shell virtual Compton amplitudes, defined by the conditions,

$$
p_{1}^{2}=p_{2}^{2}=-M_{H}^{2},
$$

depend only on four independent scalar invariants. The gauge-invariant tensors in (2.6) will be denoted respectively as $\mathcal{V}_{i}^{\mu \nu}\left(p, q_{1}, q_{2}\right), i=1, \cdots 5$.

We shall be interested more particularly in two kinematical regimes, according to whether one or two photons are real. In electrophotoproduction the outgoing photon is real, $q_{2}^{2}=0$, and thus transversely polarized. One can contract the Compton amplitude with the polarization $\epsilon_{2}$, set $\epsilon_{2} \cdot q_{2}=0$ and still impose a gauge condition on the outgoing photon, e.g. $\epsilon_{2} \cdot p=0$, by choosing the Coulomb gauge $\epsilon_{2}^{0}=0$ and the frame $\vec{p}=\mathbf{0}$. Then the contracted amplitude becomes,

$$
\begin{aligned}
A_{\mathrm{VCS}}^{\mu}=T^{\mu \nu} \epsilon_{2 \nu}^{\star}= & V_{1}\left(\epsilon_{2}^{* \mu}-\frac{q_{1}^{\mu}}{q_{1}^{2}}\left(\overrightarrow{\epsilon_{2}^{*}} \cdot \overrightarrow{q_{1}}\right)\right)+V_{3}\left(q_{2}^{\mu}-q_{1}^{\mu} \frac{\left(q_{1} \cdot q_{2}\right)}{q_{1}^{2}}\right)\left(\overrightarrow{\epsilon_{2}^{*}} \cdot \overrightarrow{q_{1}}\right) \\
& +V_{4}\left(p^{\mu}-q_{1}^{\mu} \frac{\left(p \cdot q_{1}\right)}{q_{1}^{2}}\right)\left(\overrightarrow{\epsilon_{2}^{*}} \cdot \overrightarrow{q_{1}}\right) .
\end{aligned}
$$

Therefore there are only three independent form factors when the outgoing photon is real. 
Finally if the ingoing photon is also real, $q_{1}^{2}=0$, one can contract the amplitude $A_{\mathrm{VCS}}^{\mu}$ with the polarization $\epsilon_{1}$ and impose similarly the conditions $\epsilon_{1} \cdot q_{1}=\epsilon_{1} \cdot p=0$. Hence the real Compton amplitude has in general two independent form factors,

$$
A_{\mathrm{RCS}}=\epsilon_{1}^{\mu} T_{\mu \nu} \epsilon_{2}^{\star \nu}=V_{1} \vec{\epsilon}_{1} \cdot{\overrightarrow{\epsilon_{2}}}^{\star}+V_{3}\left(\overrightarrow{\epsilon_{1}} \cdot \overrightarrow{q_{2}}\right)\left(\vec{\epsilon}^{\star} \cdot \overrightarrow{q_{1}}\right) .
$$

\section{The soft-wall model}

The AdS/CFT correspondence is based upon the fact that the isometry group of the fivedimensional (5D) anti-de Sitter space is the same as the four-dimensional conformal group $\mathrm{SO}(4,2)$. In Poincaré coordinates, the $\mathrm{AdS}_{5}$ metric reads

$$
d s^{2}=\frac{R^{2}}{z^{2}}\left(\eta_{\mu \nu} d x^{\mu} d x^{\nu}+d z^{2}\right), \quad \sqrt{-g}=\frac{R^{5}}{z^{5}},
$$

where $\eta_{\mu \nu} \equiv(-1,1,1,1)$ is the four-dimensional Minkowski metric. We shall set the curvature radius $R$ to 1 from now on.

Following [11] we introduce a massless $5 \mathrm{D}$ vector field $A_{m}(x, z)$ with a $\mathrm{U}(1)$ gauge invariance, which is dual to the electromagnetic current. The free field $A_{m}(x, z)$ must satisfy the Maxwell equations in the bulk (with no dilaton coupling, which would break the conformal invariance of the electromagnetic field),

$$
\left(\nabla_{m} F\right)^{m n}=\frac{1}{\sqrt{-g}} \partial_{m}\left(\sqrt{-g} F^{m n}\right)=0 .
$$

Choosing the linear gauge fixing condition,

$$
\partial^{\mu} A_{\mu}+z \partial_{z}\left(z^{-1} A_{z}\right)=0
$$

the general plane-wave solution of (3.2) in the space-like region, $Q^{2}=q \cdot q>0$, reads

$$
\begin{aligned}
& A_{\mu}(x, z)=\epsilon_{\mu} e^{i q \cdot x} Q z K_{1}(Q z), \\
& A_{z}(x, z)=-i \frac{(\epsilon \cdot q)}{q^{2}} e^{i q \cdot x} \partial_{z}\left(Q z K_{1}(Q z)\right),
\end{aligned}
$$

where $\epsilon_{\mu}$ is a polarization vector. The boundary condition is chosen such that the solution becomes a plane-wave on the Minkowski slice at $z=0$,

$$
\lim _{z \rightarrow 0} A_{\mu}(x, z)=\epsilon_{\mu} e^{i q \cdot x} .
$$

The boundary condition in the timelike region, $q^{2}<0$, can be obtained by analytic continuation in the $q^{2}$ variable. A crucial property of the boundary condition (3.5) is that the vector field $A_{\mu}(x, z)$ is in fact a constant plane-wave throughout the bulk of the AdS space when $q^{2}=0$.

We introduce a massive 5D scalar field $\Phi(x, z)$ which will be the dual of an operator which creates the spinless target. Following [5] the bulk scalar field is coupled to a background dilaton field $\chi(z)$ which deforms the $\mathrm{AdS}_{5}$ metric. The action which describes the propagation of $\Phi$ in this background reads

$$
S_{\Phi}=\frac{1}{2} \int d^{4} x d z \sqrt{-g} e^{-\chi}\left(g^{i j} \partial_{i} \Phi \partial_{j} \Phi+m_{S}^{2} \Phi^{2}\right),
$$


where $g$ is the $\mathrm{AdS}_{5}$ metric. The classical field equation reads

$$
\Delta_{g} \Phi \equiv \frac{e^{\chi}}{\sqrt{-g}} \partial_{i}\left(e^{-\chi} \sqrt{-g} g^{i j} \partial_{j} \Phi\right)=m_{S}^{2} \Phi .
$$

In Poincaré coordinates, the Laplacian equation becomes

$$
z^{2} \square \Phi+z^{5} e^{\chi} \partial_{z}\left(z^{-3} e^{-\chi} \partial_{z} \Phi\right)=m_{S}^{2} \Phi .
$$

Looking for a solution that is a plane-wave in Minkowski space and setting

$$
\Phi(x, z)=e^{i p \cdot x} e^{\chi(z) / 2} z^{3 / 2} \psi(z) \equiv e^{i p \cdot x} \widehat{\Phi}(z),
$$

the Laplacian equation is transformed into a Schrödinger-like equation

$$
\begin{aligned}
\frac{d^{2} \psi}{d z^{2}}-V(z) \psi & =p^{2} \psi \\
V(z) & =\frac{m_{S}^{2}+15 / 4}{z^{2}}+\frac{3}{2 z} \partial_{z} \chi+\frac{1}{4}\left(\partial_{z} \chi\right)^{2}-\frac{1}{2} \partial_{z}^{2} \chi .
\end{aligned}
$$

If the potential $V(z)$ has the right properties, and with appropriate boundary conditions, this equation has a complete set of solutions $\left\{\psi_{n}(z), n \in \mathbb{N}\right\}$ which form an orthonormal basis of the Hilbert space $\mathcal{H}$ defined by the inner product

$$
\langle\phi \mid \psi\rangle_{\mathcal{H}}=\int_{0}^{\infty} d z \phi^{\star}(z) \psi(z) .
$$

We shall assume that the dilaton background is such that $\mathcal{H}$ is well-defined. Otherwise we let the dilaton profile unspecified to be as generic as possible, except that conformal invariance in the ultraviolet requires that $\chi(z) \rightarrow 0$ for $z \rightarrow 0$.

In terms of the plane-wave solutions of the Laplacian equation (3.8), with the corresponding boundary conditions, the completeness relation takes the form

$$
\delta\left(z-z^{\prime}\right)=\sum_{n} z^{\prime-3 / 2} e^{-\chi\left(z^{\prime}\right) / 2} \widehat{\Phi}_{n}^{\star}\left(z^{\prime}\right) \widehat{\Phi}_{n}(z) z^{-3 / 2} e^{-\chi(z) / 2} .
$$

Therefore the set of classical solutions $\left\{\widehat{\Phi}_{n}(z), n \in \mathbb{N}\right\}$ form a complete orthonormal basis of the Hilbert space $\mathcal{H}_{S}$ spanned by the solutions of the Laplacian equation and defined by the inner product,

$$
\left\langle\widehat{\Phi} \mid \widehat{\Phi}^{\prime}\right\rangle_{\mathcal{H}_{S}}=\int_{0}^{\infty} d z z^{-3} e^{-\chi(z)} \widehat{\Phi}^{\star}(z) \widehat{\Phi}^{\prime}(z)
$$

Each $\widehat{\Phi}_{n}$ is a normalized eigenfunction, with appropriate boundary conditions, of the operator

$$
\widehat{H}_{S} \widehat{\Phi}_{n}=\left(z^{3} e^{\chi} \partial_{z}\left(z^{-3} e^{-\chi} \partial_{z}\right)-m_{S}^{2} z^{-2}\right) \widehat{\Phi}_{n}=-m_{n}^{2} \widehat{\Phi}_{n} .
$$

The scalar Green function $G\left(x, z ; x^{\prime}, z^{\prime}\right)$ is defined by the inhomogeneous equation

$$
\left(\Delta_{g}-m_{S}^{2}\right) G\left(x, z ; x^{\prime}, z^{\prime}\right)=\frac{e^{\chi}}{\sqrt{-g}} \delta^{4}\left(x-x^{\prime}\right) \delta\left(z-z^{\prime}\right) .
$$


Its four-dimensional Fourier transform $\widehat{G}\left(z, z^{\prime} ; p\right)$

$$
G\left(x, z ; x^{\prime}, z^{\prime}\right)=\frac{1}{(2 \pi)^{4}} \int_{-\infty}^{+\infty} d^{4} p e^{i p\left(x^{\prime}-x\right)} \widehat{G}\left(z, z^{\prime} ; p\right)
$$

satisfies the equation

$$
\widehat{H}_{S} \widehat{G}=p^{2} \widehat{G}+z^{3} e^{\chi} \delta\left(z-z^{\prime}\right) .
$$

$\widehat{G}$ has an expansion in terms of the normalized eigenfunctions satisfying the same boundary conditions,

$$
\widehat{G}\left(z, z^{\prime} ; p\right)=-\sum_{n=0}^{\infty} \frac{\widehat{\Phi}_{n}^{\star}(z) \widehat{\Phi}_{n}\left(z^{\prime}\right)}{p^{2}+m_{n}^{2}-i \epsilon} .
$$

The handling of the singularities at $p^{2}=-m_{n}^{2}$ is done with the standard Feynman prescription.

In order to complete the definition of the model we still need to specify the interaction between the bulk scalar field and the bulk U(1) field. Since we are interested in describing the electromagnetic interactions of a charged spinless hadron, we shall take a U(1) covariant coupling, $D_{n} \Phi=\partial_{n} \Phi-i e A_{n} \Phi$. Hence we shall consider the full anti-de Sitter action,

$$
S_{\mathrm{AdS}}\left[\Phi, \Phi^{*}, A^{m}\right]=\int d^{4} x d z \sqrt{-g}\left(-\frac{1}{4} F^{m n} F_{m n}+e^{-\chi}\left(\left(D^{m} \Phi\right)^{*} D_{m} \Phi+m_{S}^{2} \Phi^{*} \Phi\right)\right) .
$$

\section{Calculation of four-point functions in AdS/QCD}

The gauge/gravity correspondence relates generating functions in a strongly-coupled gauge theory to the classical supergravity partition function in the following way:

$$
\begin{aligned}
Z_{C F T}(c, \bar{c}, n+\bar{n}) & =\left\langle\exp \left(\int d^{4} x\left(n_{\mu}+\bar{n}_{\mu}\right) J^{\mu}+\bar{c} O+c O^{\dagger}\right)\right\rangle_{C F T} \\
& =\exp \left(-S_{\text {AdS }}^{c l}\left[\Phi(c), \Phi^{*}(\bar{c}), A^{m}\left(n_{\mu}+\bar{n}_{\mu}\right)\right]\right)
\end{aligned}
$$

where the 4-dimensional sources for the CFT appear as boundary conditions for the 5dimensional classical supergravity fields. Correlation functions of CFT operators can be obtained by expanding (4.1) to linear-order with respect to the sources. We shall use the prescription (4.1) as a recipe for the AdS/QCD model. The correlation function we are interested in can be obtained from the coefficient of $\bar{c} n_{\mu} \bar{n}_{\nu} c$.

In the contracted Compton amplitude, $\epsilon_{1 \mu} T^{\mu \nu} \epsilon_{2 \nu}^{*}$, the QCD operators are coupled to asymptotic states, therefore these will serve as boundary conditions for the free bulk fields $\Phi^{(0)}, \Phi^{*(0)}$, and $A_{\mu}^{(0)}$. After we express $S_{\text {AdS }}^{\text {cl }}$ in terms of free fields, it will be easy to read off the $\bar{c} n_{\mu} \bar{n}_{\nu} c$ coefficient. 
With the notations of the previous section, the equations of motion for the interacting classical bulk fields read

$$
\begin{aligned}
\frac{1}{\sqrt{-g}} \partial_{n}\left(\sqrt{-g} F^{m n}\right) & =i e e^{-\chi}\left(\Phi^{*} D^{m} \Phi-\left(D^{m} \Phi^{*}\right) \Phi\right), \\
\left(\Delta_{g}-m_{S}^{2}\right) \Phi & =V(A) \Phi=i e V_{1}(A) \Phi+e^{2} V_{2}(A) \Phi, \\
\left(\Delta_{g}-m_{S}^{2}\right) \Phi^{*} & =\bar{V}(A) \Phi^{*}=-i e V_{1}(A) \Phi^{*}+e^{2} V_{2}(A) \Phi^{*},
\end{aligned}
$$

where the linear operators $V_{1}(A)$ and $V_{2}(A)$ act on the right and read

$$
\begin{aligned}
& V_{1}(A)=\frac{e^{\chi}}{\sqrt{-g}} \partial_{m}\left(\sqrt{-g} e^{-\chi} A^{m}\right)+2 A^{m} \partial_{m}, \\
& V_{2}(A)=A^{m} A_{m} .
\end{aligned}
$$

The function $V$ can be also used to write the interaction term in $S_{\text {AdS }}$ :

$$
\begin{aligned}
S_{\mathrm{int}} & =\int d^{4} x d z \sqrt{-g} e^{-\chi}\left(i e A^{m}\left(\Phi^{*} \partial_{m} \Phi-\Phi \partial_{m} \Phi^{*}\right)+e^{2} A^{m} A_{m} \Phi^{*} \Phi\right), \\
& =\frac{1}{2} \int d^{4} x d z \sqrt{-g} e^{-\chi}\left(\Phi^{*} V(A) \Phi+\Phi \bar{V}(A) \Phi^{*}\right) .
\end{aligned}
$$

The solutions for $\Phi$ and $\Phi^{*}$ can be written as

$$
\Phi^{(*)}(y)=\Phi_{(0)}^{(*)}(y)+\int d y^{\prime} \sqrt{-g^{\prime}} e^{-\chi\left(z^{\prime}\right)} G\left(y ; y^{\prime}\right) \stackrel{(-)}{V}\left(A\left(y^{\prime}\right)\right) \Phi^{(*)}\left(y^{\prime}\right),
$$

where the free bulk fields $\Phi_{(0)}, \Phi_{(0)}^{*}$ and the Green function $G$ are respectively solutions of (3.7) and (3.18). We use the shorthand notations $y=(x, z)$ and $d y=d^{4} x d z$. We can now write $\Phi^{*} V(A) \Phi$ in terms of the free fields:

$$
\begin{aligned}
\Phi^{*} V(A) \Phi= & \left(\Phi_{(0)}^{*}(y)-i e \int d y^{\prime} \sqrt{-g^{\prime}} e^{-\chi\left(y^{\prime}\right)} G\left(y ; y^{\prime}\right) V_{1}\left(A_{(0)}\left(y^{\prime}\right)\right) \Phi_{(0)}^{*}\left(y^{\prime}\right)\right) \\
& \times\left(i e V_{1}\left(A_{(0)}(y)\right)+e^{2} V_{1}\left(A_{(1)}(y)\right)+e^{2} V_{2}\left(A_{(0)}(y)\right)\right) \\
& \times\left(\Phi_{(0)}(y)+i e \int d y^{\prime \prime} \sqrt{-g^{\prime \prime}} e^{-\chi\left(y^{\prime \prime}\right)} G\left(y ; y^{\prime \prime}\right) V_{1}\left(A_{(0)}\left(y^{\prime \prime}\right)\right) \Phi_{(0)}\left(y^{\prime \prime}\right)\right)+\mathcal{O}\left(e^{3}\right),
\end{aligned}
$$

and similarly for $\Phi \bar{V}(A) \Phi^{*}$. In $S_{\text {int }}^{c l}$, the contribution involving $A_{(0)} A_{(0)} \Phi_{(0)}^{*} \Phi_{(0)}$ appears at order $e^{2}$ :

$$
\begin{aligned}
S_{\mathrm{int}}^{c l}= & \int d y \sqrt{-g} e^{-\chi}\left(i e A^{m}\left(\Phi^{*} \partial_{m} \Phi-\Phi \partial_{m} \Phi^{*}\right)+e^{2} A^{m} A_{m} \Phi^{*} \Phi\right) \\
& +e^{2} \int d y d y^{\prime} \sqrt{-g} e^{-\chi(y)} \sqrt{-g^{\prime}} e^{-\chi\left(y^{\prime}\right)} A^{m}(y)\left\{\left(G\left(y ; y^{\prime}\right) \partial_{m} \Phi^{*}(y)\right.\right. \\
& \left.\left.-\Phi^{*}(y) \partial_{m} G\left(y ; y^{\prime}\right)\right) V_{1}\left(A\left(y^{\prime}\right)\right) \Phi\left(y^{\prime}\right)+\left(\Phi \leftrightarrow \Phi^{*}\right)\right\}+\mathcal{O}\left(e^{3}\right),
\end{aligned}
$$

where we have dropped the $(0)$ notation for clarity, and we are now dealing only with free fields. Note that the term $V_{1}\left(A_{(1)}\right)$ in (4.9) contributes also at order $e^{2}$ but we have dropped 
it since it does not contribute to $T^{\mu \nu}$, but rather to a $\left\langle\Phi^{* 2} \Phi^{2}\right\rangle$ correlator. Finally, after integrating by parts over $y^{\prime}$ one writes:

$$
\begin{aligned}
& S_{\text {int }}^{c l}= i e \int d y \sqrt{-g} e^{-\chi} A^{m}\left(\Phi^{*} \partial_{m} \Phi-\Phi \partial_{m} \Phi^{*}\right)+e^{2} \int d y \sqrt{-g} e^{-\chi} A^{m} A_{m} \Phi^{*} \Phi \\
&+e^{2} \int d y d y^{\prime} \sqrt{-g} e^{-\chi} \sqrt{-g^{\prime}} e^{-\chi^{\prime}} A^{m}(y) A^{n}\left(y^{\prime}\right) \\
& \\
&\left\{\left(\Phi^{*}(y) \partial_{m}-\left(\partial_{m} \Phi^{*}(y)\right)\right)\left(\Phi\left(y^{\prime}\right) \partial_{n}^{\prime}-\left(\partial_{n}^{\prime} \Phi\left(y^{\prime}\right)\right)\right)\right. \\
&\left.+\left(\Phi(y) \partial_{m}-\left(\partial_{m} \Phi(y)\right)\right)\left(\Phi^{*}\left(y^{\prime}\right) \partial_{n}^{\prime}-\left(\partial_{n}^{\prime} \Phi^{*}\left(y^{\prime}\right)\right)\right)\right\} G\left(y ; y^{\prime}\right)
\end{aligned}
$$

In this expression, the boundary conditions at $z=0$ of the classical fields $A^{m}$, $\Phi$, and $\Phi^{*}$ are respectively $n_{\mu}+\bar{n}_{\mu}, c$, and $\bar{c}$. These enter in a linear way in the fields, therefore the $\bar{c} n_{\mu} \bar{n}_{\nu} c$ coefficient in the expansion of (4.1) is simply obtained. After contractions, one can write

$$
\begin{aligned}
\epsilon_{\mu} T^{\mu \nu} \epsilon_{\nu}^{*}= & 2 e^{2} \int d y \sqrt{-g} e^{-\chi} A^{m}(y) A_{m}^{*}(y) \Phi^{*}(y) \Phi(y) \\
& +e^{2} \int d y d y^{\prime} \sqrt{-g} e^{-\chi} \sqrt{-g^{\prime}} e^{-\chi^{\prime}}\left(A^{m}(y) A^{* n}\left(y^{\prime}\right)+A^{* m}(y) A^{n}\left(y^{\prime}\right)\right) \\
& \times\left(\Phi(y) \partial_{m}-\left(\partial_{m} \Phi(y)\right)\right)\left(\Phi^{*}\left(y^{\prime}\right) \partial_{n}^{\prime}-\left(\partial_{n}^{\prime} \Phi^{*}\left(y^{\prime}\right)\right)\right) G\left(y ; y^{\prime}\right)
\end{aligned}
$$

where the fields $\Phi(x, z)$ and $A(x, z)$ are the plane-wave solutions defined in section 3.

In a diagrammatic representation, the first contribution in (4.12) is a contact interaction, while the second contribution contains an $s$-channel diagram (with the term $A^{m}(x, z) A^{* n}\left(x^{\prime}, z^{\prime}\right)$ ) and a $u$-channel diagram (with the term $A^{* m}(x, z) A^{n}\left(x^{\prime}, z^{\prime}\right)$ ) [36]. This is not surprising, since it is well-known that taking the classical limit of a quantum field theory is equivalent to keeping only tree diagrams in the perturbative expansion.

\section{On-shell Compton amplitude}

The $s$-channel contribution in eq. (4.12) can be written as

$$
\begin{aligned}
\epsilon^{\mu} T_{\mu \nu}^{S} \epsilon^{\star \nu}=(i e)^{2} \int d^{4} x d^{4} x^{\prime} d z d z^{\prime} z^{-3} e^{-\chi(z)} A_{k}(x, z) A_{l}^{\star}\left(x^{\prime}, z^{\prime}\right) z^{\prime-3} e^{-\chi\left(z^{\prime}\right)} \\
\times\left(\Phi_{\text {in }}(x, z) \overleftrightarrow{\partial}_{(x, z)}^{k} G\left(x, z ; x^{\prime}, z^{\prime}\right) \overleftrightarrow{\partial}_{\left(x^{\prime}, z^{\prime}\right)} \Phi_{\text {out }}^{\star}\left(x^{\prime}, z^{\prime}\right)\right),
\end{aligned}
$$

where the initial and final wave-functions of the bulk scalar fields, $\Phi_{\text {in }}$ and $\Phi_{\text {out }}$, are normalized plane-wave solutions of the Laplacian equation (3.8), whereas the bulk vector field $A_{k}$ is a normalized plane-wave solution of the Maxwell equations. The Green function $G\left(x, z ; x^{\prime}, z^{\prime}\right)$ is defined by eqs. (3.17) and (3.18). We shall introduce the short- 
hand notations,

$$
\begin{aligned}
\Phi_{\text {in }}(x, z) & =e^{i p_{1} \cdot x} \widehat{\Phi}_{i}(z), & \Phi_{\text {out }}\left(x^{\prime}, z^{\prime}\right) & =e^{i p_{2} \cdot x^{\prime}} \widehat{\Phi}_{f}\left(z^{\prime}\right), \\
A_{\mu}(x, z) & =\epsilon_{\mu} e^{i q_{1} \cdot x} A_{1}(z), & A_{z}(x, z) & =-i \frac{\epsilon \cdot q_{1}}{q_{1}^{2}} e^{i q_{1} \cdot x} \partial_{z} A_{1}(z), \\
A_{\nu}\left(x^{\prime}, z^{\prime}\right) & =\epsilon_{\nu} e^{i q_{2} \cdot x^{\prime}} A_{2}\left(z^{\prime}\right), & A_{z}^{\prime}\left(x^{\prime}, z^{\prime}\right) & =-i \frac{\epsilon \cdot q_{2}}{q_{2}^{2}} e^{i q_{2} \cdot x^{\prime}} \partial_{z^{\prime}} A_{2}\left(z^{\prime}\right), \\
A_{1}(z) & =Q_{1} z K_{1}\left(Q_{1} z\right), & A_{2}(z) & =Q_{2} z K_{1}\left(Q_{2} z\right) .
\end{aligned}
$$

A straightforward calculation yields,

$$
\begin{aligned}
T_{\mu \nu}^{s}= & (2 \pi)^{4} \delta^{(4)}\left(p_{1}+q_{1}-p_{2}-q_{2}\right) e^{2} \times\left(\left(p_{1}+k\right)_{\mu}\left(p_{2}+k\right)_{\nu} \mathcal{F}_{1}\left(q_{1}^{2}, q_{2}^{2}, s\right)\right. \\
& \left.-\frac{\left(p_{1}+k\right)_{\mu} q_{2 \nu}}{q_{2}^{2}} \mathcal{F}_{2}\left(q_{1}^{2}, q_{2}^{2}, s\right)+\frac{q_{1 \mu}\left(p_{2}+k\right)_{\nu}}{q_{1}^{2}} \mathcal{F}_{3}\left(q_{1}^{2}, q_{2}^{2}, s\right)-\frac{q_{1 \mu} q_{2 \nu}}{q_{1}^{2} q_{2}^{2}} \mathcal{F}_{4}\left(q_{1}^{2}, q_{2}^{2}, s\right)\right) .
\end{aligned}
$$

with $k=p_{1}+q_{1}=p_{2}+q_{2}, s=k^{2}$, and

$\mathcal{F}_{1}\left(q_{1}^{2}, q_{2}^{2}, k^{2}\right)=\iint d z d z^{\prime} z^{-3} e^{-\chi(z)} A_{1}(z) \widehat{\Phi}_{i}(z) \widehat{G}\left(z, z^{\prime}, k^{2}\right) \widehat{\Phi}_{f}^{\star}\left(z^{\prime}\right) A_{2}^{\star}\left(z^{\prime}\right) z^{\prime-3} e^{-\chi\left(z^{\prime}\right)}$,

$\mathcal{F}_{2}\left(q_{1}^{2}, q_{2}^{2}, k^{2}\right)=\iint d z d z^{\prime} z^{-3} e^{-\chi(z)} A_{1}(z) \widehat{\Phi}_{i}(z)\left(\widehat{G}\left(z, z^{\prime}, k^{2}\right) \overleftrightarrow{\partial}_{z^{\prime}} \widehat{\Phi}_{f}^{\star}\left(z^{\prime}\right)\right) \partial_{z^{\prime}} A_{2}^{\star}\left(z^{\prime}\right) z^{\prime-3} e^{-\chi\left(z^{\prime}\right)}$

$\mathcal{F}_{3}\left(q_{1}^{2}, q_{2}^{2}, k^{2}\right)=\iint d z d z^{\prime} z^{-3} e^{-\chi(z)} \partial_{z} A_{1}(z)\left(\widehat{\Phi}_{i}(z) \stackrel{\leftrightarrow}{\partial}_{z} \widehat{G}\left(z, z^{\prime}, k^{2}\right)\right) \widehat{\Phi}_{f}^{\star}\left(z^{\prime}\right) A_{2}^{\star}\left(z^{\prime}\right) z^{\prime-3} e^{-\chi\left(z^{\prime}\right)}$,

$\mathcal{F}_{4}\left(q_{1}^{2}, q_{2}^{2}, k^{2}\right)=\iint d z d z^{\prime} z^{-3} e^{-\chi(z)} \partial_{z} A_{1}(z)\left(\widehat{\Phi}_{i}(z) \stackrel{\leftrightarrow}{\partial} \underset{z}{G}\left(z, z^{\prime}, k^{2}\right) \overleftrightarrow{\partial}_{z^{\prime}} \widehat{\Phi}_{f}^{\star}\left(z^{\prime}\right)\right) \partial_{z^{\prime}} A_{2}^{\star}\left(z^{\prime}\right) z^{\prime-3} e^{-\chi\left(z^{\prime}\right)}$

The $u$-channel amplitude is related to the $s$-channel amplitude according to the crossing symmetry by interchanging $\mu \leftrightarrow \nu, q_{1} \leftrightarrow-q_{2}, \epsilon \leftrightarrow \epsilon^{\star}$ and $A_{1} \leftrightarrow A_{2}^{\star}$. Hence it reads

$$
\begin{aligned}
T_{\mu \nu}^{u}= & (2 \pi)^{4} \delta^{(4)}\left(p_{1}+q_{1}-p_{2}-q_{2}\right) e^{2} \times\left(\left(p_{2}+k^{\prime}\right)_{\mu}\left(p_{1}+k^{\prime}\right)_{\nu} \mathcal{F}_{1}\left(q_{2}^{2}, q_{1}^{2}, u\right)\right. \\
& \left.+\frac{q_{1 \mu}\left(p_{1}+k^{\prime}\right)_{\nu}}{q_{1}^{2}} \mathcal{F}_{2}\left(q_{2}^{2}, q_{1}^{2}, u\right)-\frac{\left(p_{2}+k^{\prime}\right)_{\mu} q_{2 \nu}}{q_{2}^{2}} \mathcal{F}_{3}\left(q_{2}^{2}, q_{1}^{2}, u\right)-\frac{q_{1 \mu} q_{2 \nu}}{q_{1}^{2} q_{2}^{2}} \mathcal{F}_{4}\left(q_{2}^{2}, q_{1}^{2}, u\right)\right),
\end{aligned}
$$

with $k^{\prime}=p_{1}-q_{2}=p_{2}-q_{1}$ and $u=k^{\prime 2}$.

Integrating by parts the partial derivative of $A_{1}$,

$\mathcal{F}_{3}\left(q_{1}^{2}, q_{2}^{2}, k^{2}\right)=-\iint d z d z^{\prime} z^{\prime-3} e^{-\chi\left(z^{\prime}\right)} A_{2}^{\star}\left(z^{\prime}\right) \widehat{\Phi}_{f}^{\star}\left(z^{\prime}\right) A_{1}(z) \partial_{z}\left(z^{-3} e^{-\chi(z)}\left(\widehat{\Phi}_{i}(z) \overleftrightarrow{\partial}_{z} \widehat{G}\left(z, z^{\prime}, k^{2}\right)\right)\right)$,

and using equations (3.8) and (3.18), one gets

$$
\begin{aligned}
& \partial_{z}\left(z^{-3} e^{-\chi(z)}\left(\widehat{\Phi}_{i}(z) \overleftrightarrow{\partial}_{z} \widehat{G}\left(z, z^{\prime}, k^{2}\right)\right)\right)= \\
& \widehat{\Phi}_{i}(z) \partial_{z}\left(z^{-3} e^{-\chi(z)} \partial_{z} \widehat{G}\left(z, z^{\prime}, k^{2}\right)\right)-\partial_{z}\left(z^{-3} e^{-\chi(z)} \partial_{z} \widehat{\Phi}_{i}(z)\right) \widehat{G}\left(z, z^{\prime}, k^{2}\right)= \\
& z^{-3} e^{-\chi}\left(k^{2}-p_{1}^{2}\right) \widehat{\Phi}_{i}(z) \widehat{G}\left(z, z^{\prime}, k^{2}\right)+\delta\left(z-z^{\prime}\right) \widehat{\Phi}_{i}(z) .
\end{aligned}
$$


Hence

$$
\mathcal{F}_{3}\left(q_{1}^{2}, q_{2}^{2}, k^{2}\right)=\left(p_{1}^{2}-k^{2}\right) \mathcal{F}_{1}\left(q_{1}^{2}, q_{2}^{2}, k^{2}\right)-\int d z z^{-3} e^{-\chi(z)} A_{1}(z) A_{2}^{\star}(z) \widehat{\Phi}_{i}(z) \widehat{\Phi}_{f}^{\star}(z) .
$$

Similarly,

$$
\begin{aligned}
\mathcal{F}_{2}\left(q_{1}^{2}, q_{2}^{2}, k^{2}\right) & =-\iint d z d z^{\prime} z^{-3} e^{-\chi(z)} A_{1}(z) \widehat{\Phi}_{i}(z) A_{2}^{\star}\left(z^{\prime}\right) \partial_{z^{\prime}}\left(z^{\prime-3} e^{-\chi\left(z^{\prime}\right)}\left(\widehat{G}\left(z, z^{\prime}, k^{2}\right) \stackrel{\leftrightarrow}{\partial_{z^{\prime}}} \widehat{\Phi}_{f}^{\star}\left(z^{\prime}\right)\right)\right), \\
& =\left(k^{2}-p_{2}^{2}\right) \mathcal{F}_{1}\left(q_{1}^{2}, q_{2}^{2}, k^{2}\right)+\int d z z^{-3} e^{-\chi(z)} A_{1}(z) A_{2}^{\star}(z) \widehat{\Phi}_{i}(z) \widehat{\Phi}_{f}^{\star}(z) .
\end{aligned}
$$

The four-point interaction amplitude reads

$$
\begin{aligned}
\epsilon^{\mu} T_{\mu \nu}^{c} \epsilon^{\star \nu}= & -2(i e)^{2} \int d^{4} x d z \sqrt{-g} e^{-\chi} \widehat{\Phi}_{i}(x, z) g^{m n} A_{m}(x, z) A_{n}^{\star}(x, z) \widehat{\Phi}_{f}^{\star}(x, z), \\
T_{\mu \nu}^{c}= & 2 e^{2}(2 \pi)^{4} \delta^{(4)}\left(p_{1}+q_{1}-p_{2}-q_{2}\right) \times \\
& \int d z z^{-3} e^{-\chi} \widehat{\Phi}_{i}(z)\left(g_{\mu \nu} A_{1}(z) A_{2}^{\star}(z)+\frac{q_{1 \mu} q_{2 \nu}}{q_{1}^{2} q_{2}^{2}} \partial_{z} A_{1}(z) \partial_{z} A_{2}^{\star}(z)\right) \widehat{\Phi}_{f}^{\star}(z) .
\end{aligned}
$$

Let

$$
\begin{aligned}
& \mathcal{C}_{1}\left(q_{1}^{2}, q_{2}^{2}\right)=\mathcal{C}_{1}\left(q_{2}^{2}, q_{1}^{2}\right)=\int d z z^{-3} e^{-\chi(z)} A_{1}(z) A_{2}^{\star}(z) \widehat{\Phi}_{i}(z) \widehat{\Phi}_{f}^{\star}(z), \\
& \mathcal{C}_{0}\left(q_{1}^{2}, q_{2}^{2}\right)=\mathcal{C}_{0}\left(q_{2}^{2}, q_{1}^{2}\right)=\int d z z^{-3} e^{-\chi(z)} \partial_{z} A_{1}(z) \partial_{z} A_{2}^{\star}(z) \widehat{\Phi}_{i}(z) \widehat{\Phi}_{f}^{\star}(z) .
\end{aligned}
$$

Setting $p=p_{1}+p_{2}$, the total amplitude can be written as

$$
\begin{aligned}
T_{\mu \nu}= & e^{2}(2 \pi)^{4} \delta^{(4)}\left(p_{1}+q_{1}-p_{2}-q_{2}\right) \\
\times\left(\left(\mathcal{F}_{1}\left(q_{1}^{2}, q_{2}^{2}, s\right)+\mathcal{F}_{1}\left(q_{2}^{2}, q_{1}^{2}, u\right)\right) \times\right. & \left(\left(p_{\mu} p_{\nu}-\frac{p \cdot q_{2}}{q_{2}^{2}} p_{\mu} q_{2 \nu}-\frac{p \cdot q_{1}}{q_{1}^{2}} p_{\nu} q_{1 \mu}\right)\right. \\
& \left.+\left(q_{1 \nu} q_{2 \mu}-\frac{q_{1} \cdot q_{2}}{q_{1}^{2}} q_{1 \mu} q_{1 \nu}-\frac{q_{1} \cdot q_{2}}{q_{2}^{2}} q_{2 \mu} q_{2 \nu}\right)\right) \\
+\left(\mathcal{F}_{1}\left(q_{1}^{2}, q_{2}^{2}, s\right)-\mathcal{F}_{1}\left(q_{2}^{2}, q_{1}^{2}, u\right)\right) \times( & \left(p_{\mu} q_{1 \nu}-\frac{q_{1} \cdot q_{2}}{q_{2}^{2}} p_{\mu} q_{2 \nu}-\frac{p \cdot q_{1}}{q_{1}^{2}} q_{1 \mu} q_{1 \nu}\right) \\
& \left.+\left(p_{\nu} q_{2 \mu}-\frac{q_{1} \cdot q_{2}}{q_{1}^{2}} p_{\nu} q_{1 \mu}-\frac{p \cdot q_{2}}{q_{2}^{2}} q_{2 \mu} q_{2 \nu}\right)\right) \\
+ & 2 \mathcal{C}_{1}\left(q_{1}^{2}, q_{2}^{2}\right)\left(g_{\mu \nu}-\frac{q_{1 \mu} q_{1 \nu}}{q_{1}^{2}}-\frac{q_{2 \mu} q_{2 \nu}}{q_{2}^{2}}\right) \\
& \left.+\left(2 \mathcal{C}_{0}\left(q_{1}^{2}, q_{2}^{2}\right)-\mathcal{F}_{4}\left(q_{1}^{2}, q_{2}^{2}, s\right)-\mathcal{F}_{4}\left(q_{2}^{2}, q_{1}^{2}, u\right)\right) \frac{q_{1 \mu} q_{2 \nu}}{q_{1}^{2} q_{2}^{2}}\right) .
\end{aligned}
$$

Comparing with the gauge-invariant tensor basis (2.6), gauge invariance holds true if, and only if,

$$
\begin{aligned}
2 \mathcal{C}_{0}\left(q_{1}^{2}, q_{2}^{2}\right)= & \mathcal{F}_{4}\left(q_{1}^{2}, q_{2}^{2}, s\right)+\mathcal{F}_{4}\left(q_{2}^{2}, q_{1}^{2}, u\right) \\
& +\left(\mathcal{F}_{1}\left(q_{1}^{2}, q_{2}^{2}, s\right)+\mathcal{F}_{1}\left(q_{2}^{2}, q_{1}^{2}, u\right)\right)\left(\left(p \cdot q_{1}\right)\left(p \cdot q_{2}\right)+\left(q_{1} \cdot q_{2}\right)^{2}\right) \\
& +\left(\mathcal{F}_{1}\left(q_{1}^{2}, q_{2}^{2}, s\right)-\mathcal{F}_{1}\left(q_{2}^{2}, q_{1}^{2}, u\right)\right)\left(p \cdot q_{1}+p \cdot q_{2}\right)\left(q_{1} \cdot q_{2}\right) \\
& +2 \mathcal{C}_{1}\left(q_{1}^{2}, q_{2}^{2}\right)\left(q_{1} \cdot q_{2}\right) .
\end{aligned}
$$


Expanding the bidirectional derivatives, the form factor $\mathcal{F}_{4}$ reads

$$
\begin{aligned}
\mathcal{F}_{4}= & \iint d z d z^{\prime} z^{-3} e^{-\chi(z)} \partial_{z} A_{1}(z) \\
& \times\left(\left(\widehat{\Phi}_{i}(z) \partial_{z} \widehat{G}-\widehat{G} \partial_{z} \widehat{\Phi}_{i}(z)\right) \partial_{z^{\prime}} \widehat{\Phi}_{f}^{\star}\left(z^{\prime}\right)-\partial_{z^{\prime}}\left(\widehat{\Phi}_{i}(z) \partial_{z} \widehat{G}-\partial_{z} \widehat{\Phi}_{i}(z) \widehat{G}\right) \widehat{\Phi}_{f}^{\star}\left(z^{\prime}\right)\right) \\
& \times \partial_{z^{\prime}} A_{2}^{\star}\left(z^{\prime}\right) z^{\prime-3} e^{-\chi\left(z^{\prime}\right)} .
\end{aligned}
$$

We first integrate by parts over $\partial_{z} A_{1}(z)$ and use equation (5.7) in the $s$-channel,

$$
\begin{aligned}
\mathcal{F}_{4}(s)= & -\iint d z d z^{\prime} A_{1}(z) \\
& \times\left(\left(z^{-3} e^{-\chi(z)}\left(s-p_{1}^{2}\right) \widehat{\Phi}_{i}(z) \widehat{G}\left(z, z^{\prime}, s\right)+\delta\left(z-z^{\prime}\right) \widehat{\Phi}_{i}(z)\right) \partial_{z^{\prime}} \widehat{\Phi}_{f}^{\star}\left(z^{\prime}\right)\right. \\
& \left.-\partial_{z^{\prime}}\left(z^{-3} e^{-\chi}\left(s-p_{1}^{2}\right) \widehat{\Phi}_{i}(z) \widehat{G}\left(z, z^{\prime}, s\right)+\delta\left(z-z^{\prime}\right) \widehat{\Phi}_{i}(z)\right) \widehat{\Phi}_{f}^{\star}\left(z^{\prime}\right)\right) \\
& \times \partial_{z^{\prime}} A_{2}^{\star}\left(z^{\prime}\right) z^{\prime-3} e^{-\chi\left(z^{\prime}\right)}, \\
= & \left(p_{1}^{2}-s\right) \iint d z d z^{\prime} z^{-3} e^{-\chi(z)} A_{1}(z) \widehat{\Phi}_{i}(z) \\
& \times\left(\widehat{G} \partial_{z^{\prime}} \widehat{\Phi}_{f}^{\star}\left(z^{\prime}\right)-\widehat{\Phi}_{f}^{\star}\left(z^{\prime}\right) \partial_{z^{\prime}} \widehat{G}\right) \partial_{z^{\prime}} A_{2}^{\star}\left(z^{\prime}\right) z^{\prime-3} e^{-\chi\left(z^{\prime}\right)} \\
& -\int d z z^{-3} e^{-\chi(z)} A_{1}(z) \widehat{\Phi}_{i}(z) \partial_{z} \widehat{\Phi}_{f}^{\star}(z) \partial_{z} A_{2}^{\star}(z) \\
& -\int d z A_{1}(z) \widehat{\Phi}_{i}(z) \partial_{z}\left(\widehat{\Phi}_{f}^{\star}(z) \partial_{z} A_{2}^{\star}(z) z^{-3} e^{-\chi(z)}\right) .
\end{aligned}
$$

We now integrate the first term by parts over $\partial_{z^{\prime}} A_{2}^{\star}\left(z^{\prime}\right)$, use again equation (5.7), and integrate by parts the third term,

$$
\begin{aligned}
\mathcal{F}_{4}(s)= & \left(p_{1}^{2}-s\right) \iint d z d z^{\prime} z^{-3} e^{-\chi(z)} A_{1}(z) \widehat{\Phi}_{i}(z) \\
& \times\left(z^{\prime-3} e^{-\chi\left(z^{\prime}\right)}\left(s-p_{2}^{2}\right) \widehat{G}\left(z, z^{\prime}, s\right)+\delta\left(z-z^{\prime}\right)\right) \widehat{\Phi}_{f}^{\star}\left(z^{\prime}\right) A_{2}^{\star}\left(z^{\prime}\right) \\
& -\int d z z^{-3} e^{-\chi(z)} A_{1}(z) \widehat{\Phi}_{i}(z) \partial_{z} \widehat{\Phi}_{f}^{\star}(z) \partial_{z} A_{2}^{\star}(z) \\
& +\int d z z^{-3} e^{-\chi(z)} \partial_{z}\left(A_{1}(z) \widehat{\Phi}_{i}(z)\right) \widehat{\Phi}_{f}^{\star}(z) \partial_{z} A_{2}^{\star}(z)
\end{aligned}
$$

Hence

$$
\begin{aligned}
\mathcal{F}_{4}(s)= & -\left(p_{1}^{2}-s\right)\left(p_{2}^{2}-s\right) \mathcal{F}_{1}(s)+\left(p_{1}^{2}-s\right) \mathcal{C}_{1}+\mathcal{C}_{0} \\
& -\int d z z^{-3} e^{-\chi(z)} A_{1}(z)\left(\widehat{\Phi}_{i}(z) \overleftrightarrow{\partial}_{z} \widehat{\Phi}_{f}^{\star}(z)\right) \partial_{z} A_{2}^{\star}(z)
\end{aligned}
$$

A similar identity holds in the $u$-channel. Noting that

$$
\begin{aligned}
\left(p \cdot q_{1}\right)\left(p \cdot q_{2}\right)+\left(q_{1} \cdot q_{2}\right)^{2} \pm\left(p \cdot q_{1}+p \cdot q_{2}\right)\left(q_{1} \cdot q_{2}\right) & =\left(p \pm q_{1}\right) \cdot q_{2} \times\left(p \pm q_{2}\right) \cdot q_{1}, \\
\left(p+q_{2}\right) \cdot q_{1} \times\left(p+q_{1}\right) \cdot q_{2} & =\left(p_{1}^{2}-s\right)\left(p_{2}^{2}-s\right), \\
\left(p-q_{2}\right) \cdot q_{1} \times\left(p-q_{1}\right) \cdot q_{2} & =\left(p_{1}^{2}-u\right)\left(p_{2}^{2}-u\right),
\end{aligned}
$$


gauge invariance is recovered when $p_{1}^{2}=p_{2}^{2}$. Indeed, then $\widehat{\Phi}_{i}(z)=\widehat{\Phi}_{f}(z)$ since they satisfy the same equation, so the last term in eq. (5.17) vanishes.

To summarize, the on-shell Compton amplitude reads, with $p_{1}^{2}=p_{2}^{2}=-m^{2}$,

$$
T^{\mu \nu}=e^{2}\left(2 \mathcal{C}_{1} \mathcal{V}_{1}^{\mu \nu}+\mathcal{C}_{+}\left(\mathcal{V}_{2}^{\mu \nu}+\mathcal{V}_{3}^{\mu \nu}\right)+\mathcal{C}_{-}\left(\mathcal{V}_{4}^{\mu \nu}+\mathcal{V}_{5}^{\mu \nu}\right)\right)
$$

where the tensors $\mathcal{V}_{i}^{\mu \nu}, i=1, \cdots 5$, are defined in eqs. (2.6) and

$$
C_{ \pm}\left(m^{2}, q_{1}^{2}, q_{2}^{2}, s, u\right)=\mathcal{F}_{1}\left(m^{2}, q_{1}^{2}, q_{2}^{2}, s\right) \pm \mathcal{F}_{1}\left(m^{2}, q_{2}^{2}, q_{1}^{2}, u\right)
$$

The $\delta$ factor expressing energy-momentum conservation is implicitly understood from now on in all formulas for the Compton amplitude. The form factors $\mathcal{F}_{1}\left(m^{2}, q_{1}^{2}, q_{2}^{2}, k^{2}\right)$ and $\mathcal{C}_{1}\left(m^{2}, q_{1}^{2}, q_{2}^{2}\right)$ are defined by

$$
\begin{aligned}
\mathcal{F}_{1}\left(m^{2}, q_{1}^{2}, q_{2}^{2}, k^{2}\right) & =\iint d z_{1} d z_{2} z_{1}^{-3} e^{-\chi\left(z_{1}\right)} A_{1}\left(z_{1}\right) \widehat{\Phi}_{i}\left(z_{1}\right) \widehat{G}\left(z_{1}, z_{2}, k^{2}\right) \widehat{\Phi}_{f}^{\star}\left(z_{2}\right) A_{2}^{\star}\left(z_{2}\right) z_{2}^{-3} e^{-\chi\left(z_{2}\right)}, \\
\mathcal{C}_{1}\left(m^{2}, q_{1}^{2}, q_{2}^{2}\right) & =\int d z z^{-3} e^{-\chi(z)} A_{1}(z) A_{2}^{\star}(z) \widehat{\Phi}_{i}(z) \widehat{\Phi}_{f}^{\star}(z)
\end{aligned}
$$

These formulas are valid for any dilaton background $\chi(z)$ on the 5D AdS space which yields a well-defined inner product on the vector space of solutions of the classical field equations. If $\chi \equiv 0$, our formulas coincide with the Compton amplitude off a dilaton calculated in [36], once we identify the normalized functions $\widehat{\Phi}_{i}$ and $\widehat{\Phi}_{f}$ with the Bessel solutions of the hard-wall model. We have shown quite generally that the gauge-invariant structure of the Compton amplitude is similar for hard-wall and soft-wall models. Note that we only get three independent Compton form factors out of a possible five. These generic properties do not depend upon the special way of breaking the conformal invariance for extending the AdS/CFT correspondence to QCD, nor upon detailed relations between Bessel functions or other special functions. It depends only upon the general structure of the classical equations satisfied by the bulk scalar fields and their Green functions in AdS space with a dilaton background.

Moreover we have shown explicitly that the off-shell Compton amplitudes $\left(p_{1}^{2} \neq p_{2}^{2}\right)$ calculated in these simplest AdS/QCD models are not gauge-invariant. This result is also not obvious and is in fact very specific to single scalar intermediate states. For example, had we allowed for non-minimal couplings with only single vector intermediate states, we would get gauge-invariant off-shell Compton amplitudes. One should perhaps emphasize that the non-gauge invariance of the off-shell four-point Compton amplitude has nothing to do with the (trivial) non-gauge invariance of the three-point function when the conformal dimensions of the initial and final scalar states are different. In our case, the initial, final, and intermediate scalar states are solutions of the same classical field equation in AdS space and have the same conformal dimension. The non-gauge invariance, when the initial and final scalar states are not the same mass eigenstates in Minkowski space, is due to the particular form of the contact term (5.10). 


\section{$6 \quad$ Deeply inelastic scattering}

The Compton amplitude (5.19) has a rather simple structure which it is enlightening to unravel. Expanding the Green function over the orthonormal eigenstates, we can write the form factor $\mathcal{F}_{1}$ in the doubly spacelike region as

$$
\begin{aligned}
\mathcal{F}_{1}\left(m^{2}, q_{1}^{2}, q_{2}^{2}, k^{2}\right) & =-\sum_{n=0}^{\infty} \frac{\Gamma\left(m^{2}, m_{n}^{2}, q_{1}^{2}\right) \Gamma^{*}\left(m^{2}, m_{n}^{2}, q_{2}^{2}\right)}{k^{2}+m_{n}^{2}-i \epsilon}, \\
\Gamma\left(m^{2}, m_{n}^{2}, Q^{2}\right) & =Q \int d z z^{-2} e^{-\chi(z)} K_{1}(Q z) \widehat{\Phi}(z) \widehat{\Phi}_{n}^{*}(z) .
\end{aligned}
$$

It is immediate to show from the AdS/QCD dictionary that the vertex function $\Gamma$ is just the unique form factor which parametrizes the most general matrix element of the conserved electromagnetic current between two (pseudo)scalar states,

$$
\left\langle p_{2}\left|J^{\mu}(0)\right| p_{1}\right\rangle=\Gamma\left(p_{1}^{2}, p_{2}^{2}, k^{2}\right)\left(p^{\mu}-\frac{p_{2}^{2}-p_{1}^{2}}{k^{2}} k^{\mu}\right), \quad p=p_{1}+p_{2}, \quad k=p_{2}-p_{1} .
$$

The electromagnetic form factor of the spinless target is defined as the elastic limit of $\Gamma$,

$$
F_{\gamma}\left(Q^{2}\right)=\Gamma\left(m^{2}, m^{2}, Q^{2}\right)=\mathcal{C}_{1}\left(m^{2}, Q^{2}, 0\right)=Q \int d z z^{-2} e^{-\chi(z)} K_{1}(Q z)\left|\widehat{\Phi}_{m}(z)\right|^{2} .
$$

Taking into account the tensorial identities

$$
\mathcal{V}_{2}^{\mu \nu}+\mathcal{V}_{3}^{\mu \nu} \pm \mathcal{V}_{4}^{\mu \nu} \pm \mathcal{V}_{5}^{\mu \nu}=\left(\left(p \pm q_{2}\right)^{\mu}-q_{1}^{\mu} \frac{\left(p \pm q_{2}\right) \cdot q_{1}}{q_{1}^{2}}\right)\left(\left(p \pm q_{1}\right)^{\nu}-q_{2}^{\nu} \frac{\left(p \pm q_{1}\right) \cdot q_{2}}{q_{2}^{2}}\right)
$$

the amplitude (5.19) can be written in a form which exhibits the tensorial structure used in [36]

$$
\begin{aligned}
T^{\mu \nu}=e^{2}\{ & \mathcal{C}_{1}\left(m^{2}, q_{1}^{2}, q_{2}^{2}\right) \mathcal{V}_{1}^{\mu \nu} \\
& -\left(2 p_{1}^{\mu}-q_{1}^{\mu} \frac{2 p_{1} \cdot q_{1}}{q_{1}^{2}}\right)\left(2 p_{2}^{\nu}-q_{2}^{\nu} \frac{2 p_{2} \cdot q_{2}}{q_{2}^{2}}\right) \sum_{n=0}^{\infty} \frac{\Gamma\left(m^{2}, m_{n}^{2}, q_{1}^{2}\right) \Gamma^{*}\left(m^{2}, m_{n}^{2}, q_{2}^{2}\right)}{s+m_{n}^{2}-i \epsilon} \\
& \left.-\left(2 p_{2}^{\mu}-q_{1}^{\mu} \frac{2 p_{2} \cdot q_{1}}{q_{1}^{2}}\right)\left(2 p_{1}^{\nu}-q_{2}^{\nu} \frac{2 p_{1} \cdot q_{2}}{q_{2}^{2}}\right) \sum_{n=0}^{\infty} \frac{\Gamma\left(m^{2}, m_{n}^{2}, q_{2}^{2}\right) \Gamma^{*}\left(m^{2}, m_{n}^{2}, q_{1}^{2}\right)}{u+m_{n}^{2}-i \epsilon}\right\} .
\end{aligned}
$$

As a by-product, the absorptive part of the forward Compton scattering amplitude reads

$$
\begin{aligned}
\operatorname{Im} T^{\mu \nu}\left(q^{2}, s\right) & =e^{2}\left(p^{\mu}+\frac{1}{x} q^{\mu}\right)\left(p^{\nu}+\frac{1}{x} q^{\nu}\right) \sum_{n=0}^{\infty} \delta\left(s+m_{n}^{2}\right)\left|\Gamma\left(m^{2}, m_{n}^{2}, q^{2}\right)\right|^{2}, \\
& \left.\approx e^{2}\left(\frac{\partial m_{n}^{2}}{\partial n}\right)^{-1}\right|_{m_{n}^{2}=-s}\left|\Gamma\left(m^{2},-s, q^{2}\right)\right|^{2}\left(p^{\mu}+\frac{1}{x} q^{\mu}\right)\left(p^{\nu}+\frac{1}{x} q^{\nu}\right), \\
q_{1} & =q_{2}=q, \quad p=2 p_{1}=2 p_{2}, \quad x=-\frac{q^{2}}{p \cdot q} .
\end{aligned}
$$


and do yield, in the hard-wall model, the same structure functions $F_{1}=0$ and $F_{2}$ as found in [11], in the large- $x$ region and in the one-particle approximation for intermediate states.

More generally, eq. (6.7) relates the scaling properties of the vertex function $\Gamma$ and of the structure function $F_{2}$ in a generic soft-wall model. Indeed the function $Q z K_{1}(Q z)$ decreases monotonically from 1 to 0 and is exponentially small at large $Q z$. Hence the $z$ dependence of the electromagnetic current can be roughly approximated as a step function of width $\mathcal{O}(1 / Q)$ and

$$
\Gamma\left(m^{2}, m_{n}^{2}, Q^{2}\right) \approx \int_{0}^{1 / Q} \frac{d z}{z^{3}} e^{-\chi(z)} \widehat{\Phi}(z) \widehat{\Phi}_{n}^{*}(z) .
$$

One can differentiate three kinematical regimes for the evaluation of the overlap integral (6.8):

- $Q^{2} \gg m^{2}, Q^{2} \gtrsim m_{n}^{2}$. For $z \lesssim 1 / Q$ and $Q$ large enough at fixed $\mathcal{Q}^{2} / m_{n}^{2}, \widehat{\Phi}(z)$ has the asymptotic behavior $\widehat{\Phi}(z) \sim z^{\Delta}$, where the conformal dimension $\Delta$ is the same as in the hard-wall model as long as $\chi(z) \rightarrow 0$ when $z \rightarrow 0$. On the other hand, since $n$ is a highly excited state, it is legitimate to use a WKB approximation for $\widehat{\Phi}_{n}(z)[24]$,

$$
\Gamma\left(m^{2}, m_{n}^{2}, Q^{2}\right) \propto C\left(m_{n}\right)\left(\frac{1}{Q}\right)^{\Delta} F\left(\frac{Q}{m_{n}}\right) .
$$

The squared normalization constant $C^{2}\left(m_{n}\right)$ of $\widehat{\Phi}_{n}(z)$ and the semiclassical density of states $\frac{\partial m_{n}^{2}}{\partial n}$ have the same dependence upon $m_{n}$. Therefore the structure function $F_{2}\left(Q^{2}, x\right)$ has the power-law scaling,

$$
\frac{x}{Q^{2}} F_{2}\left(Q^{2}, x\right) \propto\left(\frac{1}{Q^{2}}\right)^{\Delta} F^{2}(x), \quad x=\frac{Q^{2}}{Q^{2}-s} .
$$

- $Q^{2} \gg m^{2}, Q^{2} \gg m_{n}^{2}$. Then both $\widehat{\Phi}(z)$ and $\widehat{\Phi}_{n}(z)$ behave as $z^{\Delta}$ for $z \lesssim 1 / Q$. It follows that the electromagnetic form factor has the power law scaling [27],

$$
F_{\gamma}\left(Q^{2}\right) \propto\left(\frac{1}{Q^{2}}\right)^{\Delta-1}
$$

The identity of the asymptotic scaling behavior for the electromagnetic form factor and for the structure functions is a generic property of the AdS/QCD models that we consider. Such a property, which does not even depend upon whatever value of $\Delta$ is picked, is certainly difficult to reconcile with a partonic picture.

- $Q^{2} \rightarrow 0$. In that limit the overlap integral reduces to the scalar product of $\widehat{\Phi}$ and $\widehat{\Phi}_{n}$. Since the asymptotic states $\Phi_{\text {in }}(x, z)$ and $\Phi_{\text {out }}(x, z)$ must be eigenstates of the operator (3.15) with the same mass eigenvalue, all inelastic channels decouple when $Q^{2}=0$ and only the elastic channel remains opened. We shall work out some of the consequences in the next sections.

However we can already observe that there is a violation of elastic unitarity in the Compton amplitude (6.6) which is inherent to the $N_{c} \rightarrow \infty$ approximation involved 
in the AdS/QCD recipes. Indeed the total elastic Compton cross-section is of order $e^{4}$. Hence the imaginary part of the forward amplitude must vanish at order $e^{2}$ in the elastic limit to comply with the optical theorem. An absorptive part of the elastic amplitude can be generated only by loop effects which are at present beyond the reach of the AdS/QCD correspondence.

\section{$7 \quad$ Virtual Compton scattering}

When the outgoing photon is real, $q_{2}^{2}=0$, a mere inspection of eq. (2.6) shows that, in order to cancel the poles in $q_{2}^{2}$, we must have the following relations between the form factors,

$$
\begin{aligned}
V_{1}+\left(q_{1} \cdot q_{2}\right) V_{3}+\left(p \cdot q_{2}\right) V_{5} & =0 \\
\left(p \cdot q_{2}\right) V_{2}+\left(q_{1} \cdot q_{2}\right) V_{4} & =0 .
\end{aligned}
$$

Hence only three Compton form factors remain independent as already observed in section 2. However eqs. (7.1) are not manifestly satisfied by eqs. (5.19) and (5.21). We should have

$$
\begin{aligned}
2 \mathcal{C}_{1}+\left(q_{1} \cdot q_{2}\right) \mathcal{C}_{+}+\left(p \cdot q_{2}\right) \mathcal{C}_{-} & =0 \\
\left(p \cdot q_{2}\right) \mathcal{C}_{+}+\left(q_{1} \cdot q_{2}\right) \mathcal{C}_{-} & =0
\end{aligned}
$$

The second relation (7.3) reads explicitly, with the notations (5.2),

$$
\begin{aligned}
& \left(p+q_{1}\right) \cdot q_{2} \times \mathcal{F}_{1}\left(m^{2}, q_{1}^{2}, 0, s\right)+\left(p-q_{1}\right) \cdot q_{2} \times \mathcal{F}_{1}\left(m^{2}, 0, q_{1}^{2}, u\right)= \\
& \quad \iint d z_{1} d z_{2} z_{1}^{-3} e^{-\chi\left(z_{1}\right)} \widehat{\Phi}_{i}\left(z_{1}\right) \\
& \quad \times\left(-\left(p_{1}^{2}-s\right) A_{1}\left(z_{1}\right) \widehat{G}\left(z_{1}, z_{2}, s\right) A_{2}^{\star}\left(z_{2}\right)+\left(p_{2}^{2}-u\right) A_{2}^{\star}\left(z_{1}\right) \widehat{G}\left(z_{1}, z_{2}, u\right) A_{1}\left(z_{2}\right)\right) \\
& \quad \times \widehat{\Phi}_{f}^{\star}\left(z_{2}\right) z_{2}^{-3} e^{-\chi\left(z_{2}\right)}=0 .
\end{aligned}
$$

We now use the completeness relation (3.19) satisfied by the Green function $\widehat{G}$, and take into account the fact that for the electromagnetic field,

$$
\lim _{q_{2}^{2} \rightarrow 0} A_{2}(z)=1, \quad \lim _{q_{2}^{2} \rightarrow 0} \partial_{z} A_{2}(z)=0 .
$$

We integrate each term over $z_{2}$ and $z_{1}$ respectively,

$$
\begin{gathered}
\int d z_{2} z_{2}^{-3} e^{-\chi\left(z_{2}\right)} \widehat{\Phi}_{f}^{\star}\left(z_{2}\right) \widehat{\Phi}_{n}\left(z_{2}\right)=C^{\star}\left(p_{2}^{2}, m_{n}^{2}\right), \\
\int d z_{1} z_{1}^{-3} e^{-\chi\left(z_{1}\right)} \widehat{\Phi}_{i}\left(z_{1}\right) \widehat{\Phi}_{n}^{\star}\left(z_{1}\right)=C\left(p_{1}^{2}, m_{n}^{2}\right) .
\end{gathered}
$$

Hence eq. (7.3) reads

$$
\begin{aligned}
& -\left(p_{1}^{2}-s\right) \sum_{n} \frac{C^{\star}\left(p_{2}^{2}, m_{n}^{2}\right)}{m_{n}^{2}+s-i \epsilon} \int d z z^{-3} e^{-\chi(z)} \widehat{\Phi}_{i}(z) \widehat{\Phi}_{n}^{\star}(z) A_{1}(z) \\
& +\left(p_{2}^{2}-u\right) \sum_{n} \frac{C\left(p_{1}^{2}, m_{n}^{2}\right)}{m_{n}^{2}+u-i \epsilon} \int d z z^{-3} e^{-\chi(z)} \widehat{\Phi}_{f}^{\star}(z) \widehat{\Phi}_{n}(z) A_{1}(z)=0 .
\end{aligned}
$$


Using the orthogonality relations, the identity (7.3) holds true exactly only if the virtual Compton scattering is on-shell,

$$
p_{1}^{2}=p_{2}^{2}=-m_{n_{0}}^{2}, \quad \text { for some } n_{0} .
$$

By the same token eq. (7.2) reduces to the definition of $\mathcal{C}_{1}$ in (5.21),

$$
\begin{aligned}
2 \mathcal{C}_{1}\left(m^{2}, q_{1}^{2}, 0\right)+\left(p+q_{1}\right) \cdot q_{2} \times \mathcal{F}_{1}\left(m^{2}, q_{1}^{2}, 0, s\right)-\left(p-q_{1}\right) \cdot q_{2} \times \mathcal{F}_{1}\left(m^{2}, 0, q_{1}^{2}, u\right) & = \\
2 \mathcal{C}_{1}\left(m^{2}, q_{1}^{2}, 0\right)-2 \int d z z^{-3} e^{-\chi(z)}\left|\widehat{\Phi}_{m}(z)\right|^{2} A_{1}(z) & =0 .
\end{aligned}
$$

We can solve for $\mathcal{C}_{ \pm}$in terms of $\mathcal{C}_{1}$ :

$$
\begin{gathered}
\mathcal{C}_{+}=-\frac{2\left(q_{1} \cdot q_{2}\right)}{\left(q_{1} \cdot q_{2}\right)^{2}-\left(p \cdot q_{2}\right)^{2}} \mathcal{C}_{1}, \quad \mathcal{C}_{-}=\frac{2\left(p \cdot q_{2}\right)}{\left(q_{1} \cdot q_{2}\right)^{2}-\left(p \cdot q_{2}\right)^{2}} \mathcal{C}_{1}, \\
\left(q_{1} \cdot q_{2}\right)^{2}-\left(p \cdot q_{2}\right)^{2}=\left(q_{1}-p\right) \cdot q_{2} \times\left(q_{1}+p\right) \cdot q_{2}=\left(m^{2}+s\right)\left(m^{2}+u\right),
\end{gathered}
$$

The VCS amplitude has no absorptive part since $\left(m^{2}+s\right)\left(m^{2}+u\right)$ vanish only when $q_{2}=0$ owing to a non-vanishing mass $m$. Therefore the exact VCS amplitude with $q_{2}^{2}=0$ can be written as

$$
\begin{array}{r}
T_{\mu \nu}^{V C S}=e^{2} \mathcal{C}_{1}\left(m^{2}, q_{1}^{2}, 0\right)\left(2 \mathcal{V}_{1, \mu \nu}-\frac{2 m^{2}+s+u}{\left(m^{2}+s\right)\left(m^{2}+u\right)}\left(\mathcal{V}_{2, \mu \nu}+\mathcal{V}_{3, \mu \nu}\right)\right. \\
\left.+\frac{s-u}{\left(m^{2}+s\right)\left(m^{2}+u\right)}\left(\mathcal{V}_{4, \mu \nu}+\mathcal{V}_{5, \mu \nu}\right)\right)
\end{array}
$$

The unique Compton form factor reads

$$
\mathcal{C}_{1}\left(m^{2}, q_{1}^{2}, 0\right)=\int_{0}^{\infty} d z z^{-3} e^{-\chi(z)}\left|\widehat{\Phi}_{m}(z)\right|^{2} A_{1}(z),
$$

and is nothing but the electromagnetic form factor of the spinless target. Note that this formula holds in principle (for on-shell amplitudes) for any $q_{1}^{2}$, spacelike or timelike, if one can make an analytic continuation in the photon momentum.

The tensorial structure of the amplitude (7.11) is identical to point-like scalar electrodynamics in the tree level approximation except for the electromagnetic form factor which encodes all the internal structure of a spinless particle in this kind of AdS/QCD models. The threshold theorem imposes that

$$
\mathcal{C}_{1}\left(m^{2}, 0,0\right)=\int_{0}^{\infty} d z z^{-3} e^{-\chi(z)}\left|\widehat{\Phi}_{m}(z)\right|^{2}=1 .
$$

The normalization is completely fixed by electromagnetic gauge invariance and the Hilbert space structure of the classical solutions in AdS space with appropriate dilaton background.

\section{Bjorken scaling of the DVCS amplitude}

It is instructive to understand the consequences of the simplistic form of the DVCS amplitude (7.11) for a would-be dual picture in terms of partonic constituents in these kinds of AdS/QCD models. 
The Bjorken scaling of the virtual Compton form factors on a (pseudo)scalar target is usually analyzed in terms of independent gauge-invariant tensors expressed in the momenta $q=\left(q_{1}+q_{2}\right) / 2, p=p_{1}+p_{2}$ and $\Delta=p_{2}-p_{1}=q_{1}-q_{2}$ with $p_{1}^{2}=p_{2}^{2}=-M^{2}$. The four independent scalar invariant $s, u, q_{1}^{2}$ and $q_{2}^{2}$ are ordinarily traded for $Q^{2}, \Delta^{2}$, and the scaling variables $\xi$ and $\eta$ defined by

$$
Q^{2}=q^{2}, \quad \xi=-\frac{Q^{2}}{p \cdot q}, \quad \eta=-\frac{\Delta \cdot q}{p \cdot q}
$$

The large $Q^{2}$ expansion of the virtual Compton scattering amplitude on any target can be described up to twist-three, and with $q_{1}^{2}$ and $q_{2}^{2}$ arbitrary, by the tensorial structure [37]

$$
\begin{aligned}
T_{\mu \nu}^{T W 3}(q, p, \Delta)= & -\mathcal{P}_{\mu \sigma} g^{\sigma \tau} \mathcal{P}_{\tau \nu} \frac{q \cdot W_{1}}{p \cdot q}+\left(\mathcal{P}_{\mu \sigma} p^{\sigma} \mathcal{P}_{\rho \nu}+\mathcal{P}_{\mu \rho} p^{\sigma} \mathcal{P}_{\sigma \nu}\right) \frac{W_{2}^{\rho}}{p \cdot q} \\
& -\mathcal{P}_{\mu \sigma} i \epsilon^{\sigma \tau q \rho} \mathcal{P}_{\tau \nu} \frac{A_{1 \rho}}{p \cdot q}
\end{aligned}
$$

where current conservation is ensured by means of the projector

$$
\mathcal{P}_{\mu \nu}=g_{\mu \nu}-\frac{q_{2 \mu} q_{1 \nu}}{q_{1} \cdot q_{2}}
$$

and the transverse component of the momentum transfer is defined by

$$
\Delta_{\mu}^{\perp}=\Delta_{\mu}+\eta p_{\mu} .
$$

The vector $W_{2 \rho}$ depends on $W_{1 \rho}$ and $A_{1 \rho}$ by the relation $\left(\epsilon_{0123}=1\right)$,

$$
W_{2 \rho}=\xi W_{1 \rho}-\frac{\xi}{2} \frac{q \cdot W_{1}}{p \cdot q} p_{\rho}+\frac{i}{2} \frac{\epsilon_{\rho \sigma \Delta q}}{p \cdot q} A_{1 \sigma} .
$$

For a spinless target, the vectors $W_{1 \rho}$ and $A_{1 \rho}$ are defined in terms of three generalized form factors $\mathcal{H}\left(\xi, \eta, \Delta^{2}, Q^{2}\right), \mathcal{H}_{3}\left(\xi, \eta, \Delta^{2}, Q^{2}\right)$ and $\widetilde{\mathcal{H}}_{3}\left(\xi, \eta, \Delta^{2}, Q^{2}\right)$ by the relations

$$
W_{1}=\mathcal{H} p+\mathcal{H}_{3} \Delta_{\perp}, \quad A_{1 \rho}=\frac{i \epsilon_{\rho \Delta p q}}{p \cdot q} \widetilde{H}_{3} .
$$

When $q_{2}^{2}=0$, the VCS relations (7.1) are satisfied and the generalized form factors $\mathcal{H}$ 's are related to the independent form factors $V_{1}, V_{3}$ and $V_{4}$ in (2.8) as follows,

$$
\begin{aligned}
V_{1} & =-\mathcal{H} \\
V_{3} & =\frac{1}{\left(2-\frac{\eta}{\xi}\right)} \frac{1}{Q^{2}}\left(\left(1-\frac{1}{2-\frac{\eta}{\xi}}\right) \mathcal{H}-\xi \widetilde{H}_{3}\left(2-3 \frac{\eta}{\xi}\right)\right), \\
V_{4} & =\frac{-\xi}{2-\frac{\eta}{\xi}} \frac{1}{Q^{2}}\left(\mathcal{H}+2 \eta \mathcal{H}_{3}+\xi \widetilde{\mathcal{H}}_{3}\left(2-\frac{\eta}{\xi}\right)^{2}\right) \\
Q^{2} & =\frac{q_{1}^{2}}{2}\left(1-\frac{\Delta^{2}}{2 q_{1}^{2}}\right) .
\end{aligned}
$$


In perturbative $\mathrm{QCD}$, these form factors can in principle be related, through factorization, to generalized parton distributions (GPDs). However, the absence of an absorptive part in the DVCS amplitude (7.11) is difficult to accommodate with a partonic interpretation which is based on the convolution of real GPDs with coefficient functions which contain both a real and an imaginary part.

Specializing to the Bjorken limit of the DVCS amplitude (7.11),

$$
\Delta^{2}=0, \quad \xi=\eta=\frac{x_{B}}{2-x_{B}}, \quad x_{B}=-\frac{q_{1}^{2}}{2 p_{1} \cdot q_{1}},
$$

one gets for the generalized form factors

$$
\begin{aligned}
\mathcal{H} & =-2 \mathcal{C}_{1}\left(m^{2}, 2 Q^{2}, 0\right), \\
\mathcal{H}_{3} & =-\widetilde{\mathcal{H}}_{3}=\frac{\xi}{1-\xi^{2}} \mathcal{H}=\frac{x_{B}\left(2-x_{B}\right)}{4\left(1-x_{B}\right)} \mathcal{H} .
\end{aligned}
$$

Therefore the asymptotic behavior in $Q^{2}$ of the DVCS cross-section integrated over $t$ and over the azimuthal angle is governed by the power-law behavior of the electromagnetic form factor. A power-law behavior in accordance with the dimensional counting rules, e.g. a scaling dimension $\Delta=2$ for the pion, cannot be consistent with a partonic interpretation of the DVCS amplitude for spinless hadronic targets.

\section{Polarizabilities}

The structure of the VCS amplitude (7.11) and the threshold theorem, eq. (7.13), have a still more drastic consequence. Real Compton scattering on a scalar target in AdS/QCD models with minimal coupling to the photon is exactly the same as in point-like scalar electrodynamics in the tree level approximation, a fact which was observed in the hardwall model of [36]. We elaborate on the implications for AdS/QCD in this section.

The first consequence is that the static polarizabilities of the scalar target vanish. Polarizabilities give the corrections to Thompson scattering which are quadratic in the energy of the photons [38]. The amplitude for real Compton scattering off a spinless particle like the pion,

$$
\gamma\left(q_{1}\right) \pi\left(p_{1}\right) \longrightarrow \gamma\left(q_{2}\right) \pi\left(p_{2}\right)
$$

can be expanded in powers of the energies of the photons near threshold and reads, in the non-relativistic limit, $\omega_{i}^{2} \ll m_{\pi}^{2}$, in the laboratory frame and in the Coulomb gauge,

$$
A(\gamma \pi \rightarrow \gamma \pi)=2 e^{2} \overrightarrow{\epsilon_{1}} \cdot \overrightarrow{\epsilon_{2}}+8 \pi m_{\pi} \omega_{1} \omega_{2}\left(\alpha_{E} \overrightarrow{\epsilon_{1}} \cdot \overrightarrow{\epsilon_{2}}+\beta_{M}\left(\overrightarrow{\epsilon_{1}} \times \widehat{q}_{1}\right) \cdot\left(\overrightarrow{\epsilon_{2}} \times \widehat{q}_{2}\right)\right)+\cdots
$$

where $q_{i}=\omega_{i}\left(1, \widehat{q}_{i}\right)$ and $\overrightarrow{\epsilon_{i}}$ are the momentum and polarization vector of each photon (with $\left.\widehat{q}_{i}^{2}=\vec{\epsilon}_{i}^{2}=1\right) . \alpha_{E}$ and $\beta_{M}$ are the electric and magnetic polarizabilities respectively. They measure the linear response of a particle with an internal structure to a small external electromagnetic perturbation. 
The cancellations of the poles in $q_{1}^{2}=0$ and $q_{2}^{2}=0$ in the Compton tensor (2.6) impose the following relations between the Compton form factors,

$$
V_{1}+\left(q_{1} \cdot q_{2}\right) V_{3}=-\left(p \cdot q_{1}\right) V_{4}=-\left(p \cdot q_{2}\right) V_{5}=\frac{\left(p \cdot q_{1}\right)\left(p \cdot q_{2}\right)}{q_{1} \cdot q_{2}} V_{2} .
$$

The most general gauge-invariant real Compton tensor can be written in terms of the two independent form factors $V_{1}$ and $V_{2}$,

$$
T_{R C S}^{\mu \nu}=V_{1}\left(g^{\mu \nu}-\frac{q_{1}^{\nu} q_{2}^{\mu}}{q_{1} \cdot q_{2}}\right)+V_{2}\left(p^{\mu}-\frac{p \cdot q_{1}}{q_{1} \cdot q_{2}} q_{2}^{\mu}\right)\left(p^{\nu}-\frac{p \cdot q_{2}}{q_{1} \cdot q_{2}} q_{1}^{\nu}\right) .
$$

Since the static polarizabilties $\alpha_{E}$ and $\beta_{M}$ are defined in the laboratory frame, $\overrightarrow{p_{1}}=\mathbf{0}$, it is convenient to choose the Coulomb gauge and impose the conditions $\epsilon_{1} \cdot p_{1}=\epsilon_{2}^{*} \cdot p_{1}=0$. Therefore the contracted real Compton amplitude can be written as

$$
A_{\mathrm{RCS}}=\epsilon_{1}^{\mu} T_{\mu \nu} \epsilon_{2}^{\star \nu}=V_{1}{\overrightarrow{\epsilon_{1}}}_{\vec{\epsilon}_{2}}{ }^{\star}+\left(V_{3}-V_{2}\right)\left(\overrightarrow{\epsilon_{1}} \cdot \overrightarrow{q_{2}}\right)\left({\overrightarrow{\epsilon_{2}}}^{\star} \cdot \overrightarrow{q_{1}}\right)
$$

We can use the identity,

$$
\left(\overrightarrow{\epsilon_{1}} \times \overrightarrow{q_{1}}\right) \cdot\left(\overrightarrow{\epsilon_{2}} \times \overrightarrow{q_{2}}\right)=\left(\overrightarrow{\epsilon_{1}} \cdot \overrightarrow{\epsilon_{2}}\right)\left(\overrightarrow{q_{1}} \cdot \overrightarrow{q_{2}}\right)-\left(\overrightarrow{\epsilon_{1}} \cdot \overrightarrow{q_{2}}\right)\left(\overrightarrow{\epsilon_{2}} \cdot \overrightarrow{q_{1}}\right)
$$

and relate the electric and magnetic polarizabilities in the lab-frame to the Compton form factors,

$$
\begin{aligned}
8 \pi m \alpha_{E} & =\left.\frac{\partial^{2}}{\partial \omega_{1} \partial \omega_{2}}\left(V_{1}+\left(V_{3}-V_{2}\right) \overrightarrow{q_{1}} \cdot \overrightarrow{q_{2}}\right)\right|_{\omega_{1}=\omega_{2}=0} \\
8 \pi m \beta_{M} & =\left.\left(V_{2}-V_{3}\right)\right|_{\omega_{1}=\omega_{2}=0} .
\end{aligned}
$$

In the particular case of (7.11), in the limit $q_{1}^{2}=0$, we have $V_{1}=2$ and $V_{2}=V_{3}$. Hence $\alpha_{E}=\beta_{M}=0$ as expected for real Compton scattering on a structureless particle.

The point-like nature of the real Compton scattering is a direct consequence of the minimal coupling between the bulk vector field and the bulk scalar field in AdS space together with the boundary condition (3.5). In order to get non-vanishing polarizabilities we need to introduce non-minimal couplings between the bulk vector field and the bulk scalar field in anti-de Sitter space.

It is well-known that the same problematics is encountered in the calculation of the pion polarizabilities in chiral perturbation theory $(\chi \mathrm{PT})$. At lowest-order in the momentum expansion, the pion is coupled minimally to the electromagnetic field $A_{\mu}$ and the polarizabilities vanish. The chiral Lagrangian at tree evel can only predict the $\pi$ - $\pi$ scattering lengths. Only the phenomenological chiral couplings of order 4 can produce non-zero polarizabilities. It can be shown [39] that the predictions at order $p^{4}$ in the chiral limit for the electric and magnetic polarizabilities of the charged pion,

$$
\alpha_{E}=\frac{4 \alpha}{m_{\pi} F_{\pi}^{2}}\left(L_{9}^{r}+L_{10}^{r}\right), \quad \alpha_{E}+\beta_{M}=0,
$$


are generated by the four-dimensional effective Lagrangian,

$$
\begin{aligned}
& -i L_{9} F_{\mu \nu} \operatorname{Tr}\left(Q D^{\mu} \mathrm{U}\left(D^{\nu} U\right)^{\dagger}+Q\left(D^{\mu} U\right)^{\dagger} D^{\nu} U\right)+L_{10} F_{\mu \nu} F^{\mu \nu} \operatorname{Tr}\left(Q U Q U^{\dagger}\right), \\
& D_{\mu} U=\partial_{\mu} U+i e A_{\mu}[Q, U] .
\end{aligned}
$$

It is therefore very easy to write down a covariant and gauge-invariant effective action in the 5D AdS space that can be added to the minimal action (3.20) to generate non-vanishing polarizabilities at the classical level for a charged (pseudo)scalar particle, e.g.,

$$
\begin{aligned}
S_{\mathrm{AdS}}^{\prime}\left[\Phi, \Phi^{*}, A^{m}\right]=\int d^{4} x d z \sqrt{-g} e^{-\chi}\left(i g _ { 1 } \frac { e } { 2 } F _ { m n } \left(D^{m} \Phi\left(D^{n} \Phi\right)^{*}\right.\right. & \left.-\left(D^{m} \Phi\right)^{*} D^{n} \Phi\right) \\
& \left.+g_{2} \frac{e^{2}}{4} F_{m n} F^{m n} \Phi^{*} \Phi\right) .
\end{aligned}
$$

Of course one could even go one step further in phenomenology and introduce non-minimal couplings between bulk fields of various spin and parity, in the spirit of the effective Lagrangian approach.

\section{Conclusion}

We have worked within the bottom-up approach to the AdS/QCD correspondence and calculated the Compton amplitude with an arbitrary dilaton background. There is a very recent study [36], within the approach of [11], which overlaps with ours. There are however significant differences which make the two papers complementary. Working in a generic soft-wall model has helped us to clarify the Lorentz-invariant and gauge-invariant structure of the Compton amplitude predicted by AdS/QCD. Moreover the structure of the Compton amplitude does not depend upon the infrared cutoff parametrized by the dilaton background.

We have found that the minimal coupling of a bulk (pseudo)scalar field to the electromagnetic current cannot reproduce the expected low-energy behavior of the Compton amplitude off a spinless composite charged particle, and produces a too simple structure in the DVCS kinematical region for a partonic interpretation.

We have pointed out an obvious signature of this failure, namely the vanishing of the electric and magnetic polarizabilities of the scalar target. The experimental situation is rather unsatisfactory since the extraction of the experimental values is model dependent. For instance the most recent experimental values for the polarizabilities of the charged pion are,

$$
\begin{aligned}
\left(\alpha_{E}-\beta_{M}\right)_{\pi^{+}} & =\left(11.6 \pm 1.5_{\text {stat }} \pm 3.0_{\text {syst }} \pm 0.5_{\text {mod }}\right) \times 10^{-4} \mathrm{fm}^{3} \quad[34] \\
\left(\alpha_{E}=-\beta_{M}\right)_{\pi^{+}} & =\left(2.5 \pm 1.7_{\text {stat }} \pm 0.6_{\text {syst }}\right) \times 10^{-4} \mathrm{fm}^{3} \quad[35] .
\end{aligned}
$$

Even if these values are still imprecise, the inclusion of non-minimal couplings to the photon is certainly required to obtain a realistic description of Compton scattering in AdS/QCD at the classical level. For instance, we note that non-minimal couplings to vector 
fields generate five independent Compton form factors, (as opposed to only three with the minimal coupling we considered in this paper), as allowed by gauge and Lorentz invariance.

Such couplings appear naturally in chiral perturbation theory. Besides, an algebra of currents based on chiral symmetry is the standard framework to describe the hadronic electromagnetic current. The AdS/QCD models we have examined do not incorporate chiral flavor symmetry nor vector meson dominance. There are several variants of chiral AdS/QCD models [40-43] and it is not the purpose of the present work to commit to one of them. Nethertheless we have identified a bare-bones effective anti-de Sitter action that can contribute to the polarizabilities in many chiral models.

In any case it should now be clear that the calculation, and the precise measurement, of the hadronic polarizabilities is a selective testing ground for the AdS/QCD correspondence.

\section{Acknowledgments}

We wish especially to thank B. Pire for inspiring discussions and very useful comments about the manuscript. We also wish to thank V. Bernard, J.P. Lansberg, B. Moussallam, T.N. Pham, U. Reinosa, L. Szymanowski and B. Xiao for very interesting discussions. C.M. is supported by the European Commission under the FP6 program, contract No. MOIF-CT-2006-039860. This work is partly supported by the ANR-06-JCJC-0084.

\section{A Explicit formulas in the hard-wall model}

The hard-wall model is defined by the absence of dilaton background, $\chi(z)=0$, and by imposing a Dirichlet boundary condition on the massive fields at some finite cutoff in $z$. Then the plane-wave solution for a massive scalar field reads (in the timelike region),

$$
\Phi(x, z)=C_{\Delta-1}(m) e^{i p \cdot x} z^{2} J_{\Delta-2}(m z), \quad \Delta=2 \pm \sqrt{m_{S}^{2}+4} \geq 1, \quad p^{2}=-m^{2}<0,
$$

(this is not the most general admissible solution for $1 \leq \Delta \leq 3$ ). The normalization constants,

$$
C_{\Delta-1}(m)=\sqrt{2} \Lambda J_{\Delta-1}^{-1}\left(\frac{m}{\Lambda}\right)
$$

are defined by requiring,

$$
\int_{0}^{1 / \Lambda} d z z^{-3}|\Phi(x, z)|^{2}=1, \quad J_{\Delta-2}\left(\frac{m}{\Lambda}\right)=0 .
$$

The scalar propagator reads,

$$
\begin{aligned}
\widehat{G}\left(z_{1}, z_{2},-m^{2}\right) & =\sum_{n} C_{\Delta-1}^{2}\left(m_{n}\right) \frac{z_{1}^{2} J_{\Delta-2}\left(m_{n} z_{1}\right) z_{2}^{2} J_{\Delta-2}\left(m_{n} z_{2}\right)}{m^{2}-m_{n}^{2}+i \epsilon} \\
m_{n} & =\zeta_{\Delta-2, n} \Lambda
\end{aligned}
$$


where $\zeta_{\nu, n}$ are the zeroes of the Bessel function $J_{\nu}(z)$. When $\Lambda \rightarrow 0$, the scalar propagator becomes,

$$
\widehat{G}\left(z_{1}, z_{2},-m^{2}\right) \underset{\Lambda \rightarrow 0}{\approx}\left(z_{1} z_{2}\right)^{2} \int_{0}^{\infty} d \mu \mu \frac{J_{\Delta-2}\left(\mu z_{1}\right) J_{\Delta-2}\left(\mu z_{2}\right)}{m^{2}-\mu^{2}+i \epsilon}+\mathcal{O}(\Lambda) .
$$

Plugging the explicit wave-functions into (7.12) one gets for the scalar DVCS form factor,

$$
\mathcal{C}_{1}\left(m^{2}, Q^{2}, 0\right)=C_{\Delta-1}^{2}(m) Q \int_{0}^{1 / \Lambda} d z z^{2} J_{\Delta-2}^{2}(m z) K_{1}(Q z) .
$$

As long as $\Delta>1$ and $Q \gg \Lambda$, we can set $\Lambda=0$ in the integration domain and use the integral formula,

$$
\mathcal{C}_{1}\left(m^{2}, Q^{2}, 0\right) \simeq 2(\Delta-1) \frac{C_{\Delta-1}^{2}(m)}{m^{2}} \times\left(\frac{m^{2}}{Q^{2}}\right)^{\Delta-1} \times \frac{(1-w)^{2 \Delta}}{\left(1-w^{2}\right)^{2}}\left(1+\frac{1}{\Delta-1} \frac{2 w^{2}}{1-w^{2}}\right)
$$

where $w$ is defined by

$$
w=1+\frac{Q^{2}}{2 m^{2}}-\sqrt{\left(1+\frac{Q^{2}}{2 m^{2}}\right)^{2}-1}
$$

Noting that

$$
w \underset{Q^{2} \rightarrow \infty}{\approx} \frac{m^{2}}{Q^{2}}\left(1+\mathcal{O}\left(\frac{m^{2}}{Q^{2}}\right)\right) \longrightarrow 0
$$

we obtain the leading large $Q^{2}$ behavior of the DVCS form factor found in [36],

$$
\mathcal{C}_{1}\left(m^{2}, Q^{2}, 0\right)=2(\Delta-1) \frac{C_{\Delta-1}^{2}(m)}{m^{2}} \times\left(\frac{m^{2}}{Q^{2}}\right)^{\Delta-1} .
$$

Open Access. This article is distributed under the terms of the Creative Commons Attribution Noncommercial License which permits any noncommercial use, distribution, and reproduction in any medium, provided the original author(s) and source are credited.

\section{References}

[1] J.M. Maldacena, The large-N limit of superconformal field theories and supergravity, Adv. Theor. Math. Phys. 2 (1998) 231 [Int. J. Theor. Phys. 38 (1999) 1113] [hep-th/9711200] [SPIRES].

[2] E. Witten, Anti-de Sitter space and holography, Adv. Theor. Math. Phys. 2 (1998) 253 [hep-th/9802150] [SPIRES].

[3] S.S. Gubser, I.R. Klebanov and A.M. Polyakov, Gauge theory correlators from non-critical string theory, Phys. Lett. B 428 (1998) 105 [hep-th/9802109] [SPIRES].

[4] J. Polchinski and M.J. Strassler, Hard scattering and gauge/string duality, Phys. Rev. Lett. 88 (2002) 031601 [hep-th/0109174] [SPIRES]. 
[5] O.J. Rosten, A Manifestly Gauge Invariant and Universal Calculus for SU(N) Yang-Mills, Int. J. Mod. Phys. A 21 (2006) 4627 [hep-th/0602229] [SPIRES].

[6] H. Boschi-Filho and N.R.F. Braga, Gauge/string duality and scalar glueball mass ratios, JHEP 05 (2003) 009 [hep-th/0212207] [SPIRES].

[7] G.F. de Teramond and S.J. Brodsky, The hadronic spectrum of a holographic dual of QCD, Phys. Rev. Lett. 94 (2005) 201601 [hep-th/0501022] [SPIRES].

[8] T. Hambye, B. Hassanain, J. March-Russell and M. Schvellinger, Four-point functions and kaon decays in a minimal AdS/QCD model, Phys. Rev. D 76 (2007) 125017 [hep-ph/0612010] [SPIRES].

[9] P. Colangelo, F. De Fazio, F. Giannuzzi, F. Jugeau and S. Nicotri, Light scalar mesons in the soft-wall model of AdS/QCD, Phys. Rev. D 78 (2008) 055009 [arXiv:0807.1054] [SPIRES].

[10] J. Erlich, How Well Does AdS/QCD Describe QCD?, Int. J. Mod. Phys. A 25 (2010) 411 [arXiv:0908.0312] [SPIRES].

[11] J. Polchinski and M.J. Strassler, Deep inelastic scattering and gauge/string duality, JHEP 05 (2003) 012 [hep-th/0209211] [SPIRES].

[12] Y. Hatta, E. Iancu and A.H. Mueller, Deep inelastic scattering at strong coupling from gauge/string duality: the saturation line, JHEP 01 (2008) 026 [arXiv:0710.2148] [SPIRES].

[13] C.A. Ballon Bayona, H. Boschi-Filho and N.R.F. Braga, Deep inelastic scattering from gauge string duality in the soft wall model, JHEP 03 (2008) 064 [arXiv:0711.0221] [SPIRES].

[14] C.A. Ballon Bayona, H. Boschi-Filho and N.R.F. Braga, Deep inelastic structure functions from supergravity at small $x$, JHEP 10 (2008) 088 [arXiv:0712.3530] [SPIRES].

[15] L. Cornalba and M.S. Costa, Saturation in Deep Inelastic Scattering from AdS/CFT, Phys. Rev. D 78 (2008) 096010 [arXiv:0804.1562] [SPIRES].

[16] Y. Hatta and T. Matsuo, Thermal hadron spectrum in $e^{+} e^{-}$annihilation from gauge/string duality, Phys. Rev. Lett. 102 (2009) 062001 [arXiv:0807.0098] [SPIRES].

[17] Y. Hatta and T. Matsuo, Thermal hadron spectrum in $e^{+} e^{-}$annihilation from gauge/string duality, Phys. Rev. Lett. 102 (2009) 062001 [arXiv:0807. 0098] [SPIRES].

[18] C.A. Ballon Bayona, H. Boschi-Filho and N.R.F. Braga, Deep inelastic scattering from gauge string duality in D3-D7 brane model, JHEP 09 (2008) 114 [arXiv:0807.1917] [SPIRES].

[19] J.-H. Gao and B.-W. Xiao, Polarized Deep Inelastic and Elastic Scattering From Gauge/String Duality, Phys. Rev. D 80 (2009) 015025 [arXiv:0904.2870] [SPIRES].

[20] Y. Hatta, T. Ueda and B.-W. Xiao, Polarized DIS in N=4 SYM: Where is spin at strong coupling?, JHEP 08 (2009) 007 [arXiv: 0905.2493] [SPIRES].

[21] E. Levin and I. Potashnikova, Soft interaction at high energy and $N=4 S Y M$, JHEP 06 (2009) 031 [arXiv:0902.3122] [SPIRES].

[22] L. Cornalba, M.S. Costa and J. Penedones, AdS black disk model for small-x DIS, arXiv: 1001.1157 [SPIRES].

[23] Y.V. Kovchegov, Z. Lu and A.H. Rezaeian, Comparing AdS/CFT Calculations to HERA $F_{2}$ Data, Phys. Rev. D 80 (2009) 074023 [arXiv: 0906.4197] [SPIRES].

[24] B. Pire, C. Roiesnel, L. Szymanowski and S. Wallon, On AdS/QCD correspondence and the partonic picture of deep inelastic scattering, Phys. Lett. B 670 (2008) 84 [arXiv:0805.4346] [SPIRES]. 
[25] H.R. Grigoryan and A.V. Radyushkin, Form Factors and Wave Functions of Vector Mesons in Holographic QCD, Phys. Lett. B 650 (2007) 421 [hep-ph/0703069] [SPIRES].

[26] H.R. Grigoryan and A.V. Radyushkin, Structure of Vector Mesons in Holographic Model with Linear Confinement, Phys. Rev. D 76 (2007) 095007 [arXiv: 0706.1543] [SPIRES].

[27] S.J. Brodsky and G.F. de Teramond, Light-Front Dynamics and AdS/QCD Correspondence: The Pion Form Factor in the Space- and Time-Like Regions, Phys. Rev. D 77 (2008) 056007 [arXiv:0707.3859] [SPIRES].

[28] H.J. Kwee and R.F. Lebed, Pion Form Factor in Improved Holographic QCD Backgrounds, Phys. Rev. D 77 (2008) 115007 [arXiv:0712.1811] [SPIRES].

[29] Z. Abidin and C.E. Carlson, Gravitational Form Factors of Vector Mesons in an AdS/QCD Model, Phys. Rev. D 77 (2008) 095007 [arXiv:0801.3839] [SPIRES].

[30] D. Rodriguez-Gomez and J. Ward, Electromagnetic form factors from the fifth dimension, JHEP 09 (2008) 103 [arXiv:0803.3475] [SPIRES].

[31] F.E. Low, Scattering of light of very low frequency by systems of spin 1/2, Phys. Rev. 96 (1954) 1428 [SPIRES].

[32] M. Gell-Mann and M.L. Goldberger, Scattering of low-energy photons by particles of spin 1/2, Phys. Rev. 96 (1954) 1433 [SPIRES].

[33] J. Portoles and M.R. Pennington, Theoretical predictions for pion polarizabilities, hep-ph/9407295 [SPIRES].

[34] J. Ahrens et al., Measurement of the $\pi^{+}$meson polarizabilities via the gamma $p \rightarrow$ gamma $\pi^{+} n$ reaction, Eur. Phys. J. A 23 (2005) 113 [nucl-ex/0407011] [SPIRES].

[35] COMPASS collaboration, A. Guskov, Pion polarizabilities measurement at COMPASS, Fizika B 17 (2008) 313 [SPIRES].

[36] J.-H. Gao and B.-W. Xiao, Non-forward Compton scattering in AdS/CFT, Phys. Rev. D 81 (2010) 035008 [arXiv:0912.4333] [SPIRES].

[37] A.V. Belitsky, D. Mueller, A. Kirchner and A. Schafer, Twist-three analysis of photon electroproduction off pion, Phys. Rev. D 64 (2001) 116002 [hep-ph/0011314] [SPIRES].

[38] V.A. Petrunkin, Scattering of Low-energy photons on a zero-spin particle, Nucl. Phys. 55 (1964) 197.

[39] J.F. Donoghue and B.R. Holstein, Pion transitions and models of chiral symmetry, Phys. Rev. D 40 (1989) 2378 [SPIRES].

[40] T. Sakai and S. Sugimoto, Low energy hadron physics in holographic QCD, Prog. Theor. Phys. 113 (2005) 843 [hep-th/0412141] [SPIRES].

[41] J. Erlich, E. Katz, D.T. Son and M.A. Stephanov, QCD and a Holographic Model of Hadrons, Phys. Rev. Lett. 95 (2005) 261602 [hep-ph/0501128] [SPIRES].

[42] L. Da Rold and A. Pomarol, Chiral symmetry breaking from five dimensional spaces, Nucl. Phys. B 721 (2005) 79 [hep-ph/0501218] [SPIRES].

[43] J. Hirn and V. Sanz, Interpolating between low and high energy QCD via a 5D Yang-Mills model, JHEP 12 (2005) 030 [hep-ph/0507049] [SPIRES]. 